\title{
THE FIRST COEFFICIENTS OF THE ASYMPTOTIC EXPANSION OF THE BERGMAN KERNEL OF THE $\operatorname{spin}^{c}$ DIRAC OPERATOR
}

\author{
XIAONAN MA AND GEORGE MARINESCU
}

\begin{abstract}
We establish the existence of the asymptotic expansion of the Bergman kernel associated to the $\operatorname{spin}^{c}$ Dirac operators acting on high tensor powers of line bundles with non-degenerate mixed curvature (negative and positive eigenvalues) by extending [15]. We compute the second coefficient $b_{1}$ in the asymptotic expansion using the method of [24].
\end{abstract}

\section{INTRODUCTION}

In [15, Theorem 4.18'], Dai, Liu and Ma established the full off-diagonal asymptotic expansion of the Bergman kernel of the $\operatorname{spin}^{c}$ Dirac operator on high tensor powers of a line bundle on a compact symplectic manifold. This paper is a continuation of their work.

For some applications, it is necessary to compute the first two coefficients $\boldsymbol{b}_{0}, \boldsymbol{b}_{1}$ of the asymptotic expansion. The approach of [15] is to relate the heat kernel and the Bergman kernel expansions. The computation of the coefficients of the Bergman kernel expansion is done by using the corresponding coefficients of the heat kernel. Thus, $b_{0}$ is calculated in [15, Theorem 1.1] and $b_{1}$ in [15, Theorem 1.3], the latter only in the Kähler case. These results brought a new proof of [14, 22, 27, 28].

Considering the symplectic case, the coefficient of $t^{1}$ in the Taylor expansion of the rescaled operator does not vanish, thus it is complicate to compute $b_{1}$ in this way. However, we developed in [24, $\S 1.5]$ a method of formal power series to compute the coefficients for the renormalized Bochner-Laplacian. The main result of this paper is Theorem 2.1, where we compute the coefficient $b_{1}$ in the asymptotic expansion of the Bergman kernel associated to the $\operatorname{spin}^{c}$ Dirac operators by applying the method in [24, $\S 1.5,2.3]$. Comparing with [24, $\S 2.3]$, the contribution from the coefficient of $t^{1}$ in the Taylor expansion of the rescaled operator $L_{2}^{t}$ in (2.7) is quite complicate here. But after taking the trace in (2.2), we refind the Hermitian scalar curvature of [17]. For more details on our approach we also refer the readers to our recent book [25].

Another feature of the paper is that we do not suppose that the almost complex structure polarizes the curvature of the line bundle, that is we allow bundles with mixed curvature (negative and positive eigenvalues). In Section[1, we explain that the arguments in [15] still work well under this condition (cf. (1.6)). Then we compute the coefficient $b_{1}$ in Section 2 ,

Date: September 14, 2018. 
Aknowledgements. We thank Professor Johannes Sjöstrand for useful conversations and the referee for useful comments.

\section{Bergman KeRnel OF THE SPIN ${ }^{c}$ DiRAC OPERATOR}

This Section is organized as follows. In Section 1.1, we recall the Lichnerowicz formula for the $\operatorname{spin}^{c}$ Dirac operator $D_{p}$. As a consequence we exhibit the spectral gap for $D_{p}^{2}$ without assumption that the almost complex structure $J$ polarizes the symplectic form $\omega$. This is done in Section 1.2. We explain in Section 1.3 the full off-diagonal asymptotic expansion for the Bergman kernel. Then we show how to handle the operator $\bar{\partial}+\bar{\partial}^{*}$, which is the content of Section 1.4, we explain also its relation to the tangent Cauchy-Riemann complex in Section 1.5 .

1.1. The $\operatorname{spin}^{c}$ Dirac operator. Let $(X, \omega)$ be a compact connected symplectic manifold of real dimension $2 n$. Assume that there exists a Hermitian line bundle $L$ over $X$ endowed with a Hermitian connection $\nabla^{L}$ with the property that $\frac{\sqrt{-1}}{2 \pi} R^{L}=$ $\omega$, where $R^{L}=\left(\nabla^{L}\right)^{2}$ is the curvature of $\left(L, \nabla^{L}\right)$. Let $\left(E, h^{E}\right)$ be a Hermitian vector bundle on $X$ with Hermitian connection $\nabla^{E}$ and its curvature $R^{E}$.

Let $J$ be an almost complex structure on $T X$ and $g^{T X}$ be a Riemannian metric on $X$ compatible with $J$, i.e. $g^{T X}(\cdot, \cdot)=g^{T X}(J \cdot, J \cdot)$. We designate by $\nabla^{T X}$ the Levi-Civita connection on $\left(T X, g^{T X}\right)$ and by $R^{T X}$ and $r^{X}$ its curvature and scalar curvature, respectively.

The almost complex structure $J$ induces a splitting $T X \otimes_{\mathbb{R}} \mathbb{C}=T^{(1,0)} X \oplus T^{(0,1)} X$, where $T^{(1,0)} X$ and $T^{(0,1)} X$ are the eigenbundles of $J$ corresponding to the eigenvalues $\sqrt{-1}$ and $-\sqrt{-1}$ respectively. Then $P^{(1,0)}=\frac{1}{2}(1-\sqrt{-1} J)$ is the projection from $T X \otimes_{\mathbb{R}} \mathbb{C}$ onto $T^{(1,0)} X$. For any $v \in T X \otimes_{\mathbb{R}} \mathbb{C}$ with decomposition $v=$ $v_{1,0}+v_{0,1} \in T^{(1,0)} X \oplus T^{(0,1)} X$, let $\bar{v}_{1,0}^{*} \in T^{*(0,1)} X$ be the metric dual of $v_{1,0}$. Then $c(v)=\sqrt{2}\left(\bar{v}_{1,0}^{*} \wedge-i_{v_{0,1}}\right)$ defines the Clifford action of $v$ on $\Lambda\left(T^{*(0,1)} X\right)$, where $\wedge$ and $i$ denote the exterior and interior product respectively.

Let $\nabla^{T^{(1,0)} X}=P^{(1,0)} \nabla^{T X} P^{(1,0)}$ be the connection on $T^{(1,0)} X$ induced by $\nabla^{T X}$, with curvature $R^{T^{(1,0)} X}$. Let $\nabla^{\text {Cliff }}$ be the Clifford connection on $\Lambda\left(T^{*(0,1)} X\right)$ induced canonically by $\nabla^{T X}$ (cf. $\left.[23, \S 2]\right)^{1}$ and let $R^{\text {Cliff }}$ be its curvature. Let $\left\{e_{i}\right\}_{1 \leqslant i \leqslant 2 n}$ be an orthonormal basis of $T X$. From the definitions, we get (cf. also [24, (0.12)])

$$
\begin{aligned}
& R^{T^{(1,0)} X}=P^{1,0}\left[R^{T X}-\frac{1}{4}\left(\nabla^{X} J\right) \wedge\left(\nabla^{X} J\right)\right] P^{1,0}, \\
& R^{\mathrm{Cliff}}=\frac{1}{4}\left\langle R^{T X} e_{l}, e_{m}\right\rangle c\left(e_{l}\right) c\left(e_{m}\right)+\frac{1}{2} \operatorname{Tr}\left[R^{T^{(1,0)} X}\right] .
\end{aligned}
$$

Let $\nabla^{E_{p}}$ be the connection on $E_{p}:=\Lambda\left(T^{*(0,1)} X\right) \otimes L^{p} \otimes E$ induced by $\nabla^{\text {Cliff }}, \nabla^{L}$ and $\nabla^{E}$. The $\operatorname{spin}^{c}$ Dirac operator $D_{p}$ is defined using $\nabla^{E_{p}}$ and acts on $\Omega^{0, \bullet}\left(X, L^{p} \otimes E\right)=$ $\bigoplus_{q=0}^{n} \Omega^{0, q}\left(X, L^{p} \otimes E\right)$, the direct sum of spaces of $(0, q)$-forms with values in $L^{p} \otimes E$.

\footnotetext{
${ }^{1}$ In [23, (2.3)], one missed a term ' $+\frac{1}{2} \operatorname{Tr}_{T^{(1,0)} X} \Gamma^{\prime}$ in the right hand side of the first line, and the second line should be read as ${ }^{\prime}=d+\sum_{l m}\left\{\left\langle\Gamma w_{l}, \bar{w}_{m}\right\rangle \bar{w}^{m} \wedge i_{\bar{w}_{l}}+^{\prime}\right.$
} 
We refer the reader to [23, (2.6)] for the precise definition. We content ourselves to describe the operator $D_{p}^{2}$ in the sequel.

Let $\langle\cdot, \cdot\rangle_{E_{p}}$ be the metric on $E_{p}$ induced by $g^{T X}, h^{L}$ and $h^{E}$. Let $d v_{X}$ be the Riemannian volume form of $\left(T X, g^{T X}\right)$. The $L^{2}$-scalar product on $\Omega^{0, \bullet}\left(X, L^{p} \otimes E\right)$, the space of smooth sections of $E_{p}$, is given by

$$
\left\langle s_{1}, s_{2}\right\rangle=\int_{X}\left\langle s_{1}(x), s_{2}(x)\right\rangle_{E_{p}} d v_{X}(x) .
$$

We denote the corresponding norm with $\|\cdot\|_{L^{2}}$.

Let $\left(\nabla^{E_{p}}\right)^{*}$ be the formal adjoint of $\nabla^{E_{p}}$ with respect to (1.2). Set

$$
\mathbf{c}(R)=\frac{1}{2}\left(R^{E}+\frac{1}{2} \operatorname{Tr}\left[R^{T^{(1,0)}} X\right)\left(e_{l}, e_{m}\right) c\left(e_{l}\right) c\left(e_{m}\right) .\right.
$$

Then the Lichnerowicz formula [4, Theorem 3.52] (cf. [23, Theorem 2.2]) for $D_{p}^{2}$ is

$$
D_{p}^{2}=\left(\nabla^{E_{p}}\right)^{*} \nabla^{E_{p}}+\frac{1}{4} r^{X}+\frac{1}{2} p R^{L}\left(e_{l}, e_{m}\right) c\left(e_{l}\right) c\left(e_{m}\right)+\mathbf{c}(R) .
$$

The Bergman kernel $P_{p}\left(x, x^{\prime}\right),\left(x, x^{\prime} \in X\right)$, is the smooth kernel with respect to $d v_{X}\left(x^{\prime}\right)$ of the orthogonal projection $P_{p}$ from $\Omega^{0, \bullet}\left(X, L^{p} \otimes E\right)$ on Ker $D_{p}$. Then $P_{p}(x, x)$ is an element of $\operatorname{End}\left(\Lambda\left(T^{*(0,1)} X\right) \otimes E\right)_{x}$.

1.2. Spectral gap of the $\operatorname{spin}^{c}$ Dirac operator. We choose the almost complex structure $J$ such that $\omega$ is $J$-invariant, i.e. $\omega(\cdot, \cdot)=\omega(J \cdot, J \cdot)$. But we do not suppose that $\omega(\cdot, J \cdot)$ is positive in Sections 1.2 1.4. This is the difference comparing with the assumption in [23, 15, 24].

Let $\mathbf{J}: T X \longrightarrow T X$ be the skew-adjoint linear map which satisfies the relation

$$
\omega(u, v)=g^{T X}(\mathbf{J} u, v)
$$

for $u, v \in T X$. Then $J$ commutes with $\mathbf{J}$. Thus $\mathbf{J} \in \operatorname{End}\left(T^{(1,0)} X\right)$, and for any $x \in X$, we can diagonalize $\mathbf{J}_{x}$, i.e. find an orthonormal basis $\left\{w_{j}\right\}_{j=1}^{n}$ of $T^{(1,0)} X$ such that $\mathbf{J}_{x} w_{j}=\frac{\sqrt{-1}}{2 \pi} a_{j}(x) w_{j}$ with $a_{j}(x) \in \mathbb{R}$. As $\omega$ is non-degenerate, the number of negative eigenvalue of $\mathbf{J}_{x} \in \operatorname{End}\left(T_{x}^{(1,0)} X\right)$ does not depend on $x$, and we denote it by $q$ (In [23, 15, 24], we suppose that $q=0$ ). From now on, we assume that

$$
\mathbf{J}_{x} w_{j}=\frac{\sqrt{-1}}{2 \pi} a_{j} w_{j}, \quad a_{j}(x)<0 \text { for } j \leqslant q \text { and } a_{j}(x)>0 \text { for } j>q .
$$

Then the vectors $\left\{w_{j}\right\}_{j=1}^{q}$ span a sub-bundle $W$ of $T^{(1,0)} X$. Set

$$
\begin{aligned}
& \omega_{d}(x)=-\sum_{j=1}^{n} a_{j} \bar{w}^{j} \wedge i_{\bar{w}_{j}}+\sum_{j=1}^{q} a_{j}=\sum_{j=1}^{q} a_{j} i_{\bar{w}_{j}} \wedge \bar{w}^{j}-\sum_{j=q+1}^{n} a_{j} \bar{w}^{j} \wedge i_{\bar{w}_{j}}, \\
& \tau(x)=\pi \operatorname{Tr}\left|T X\left(-\mathbf{J}^{2}\right)^{1 / 2}=\sum_{j=1}^{n}\right| a_{j} \mid=-\sum_{j=1}^{q} a_{j}+\sum_{j=q+1}^{n} a_{j}, \\
& \mu_{0}=\inf _{x \in X, j}\left|a_{j}(x)\right| .
\end{aligned}
$$


Then we have

$$
\frac{1}{2} R^{L}\left(e_{l}, e_{m}\right) c\left(e_{l}\right) c\left(e_{m}\right)=-2 \omega_{d}-\tau \text {. }
$$

The following result extends [23, Theorem 2.5] to the current situation. We denote by $\Omega^{\neq q}\left(X, L^{p} \otimes E\right)=\bigoplus_{k \neq q} \Omega^{0, k}\left(X, L^{p} \otimes E\right)$.

Theorem 1.1. There exists $C>0$ such that for any $p \in \mathbb{N}$

$$
\left\|D_{p} s\right\|_{L^{2}}^{2} \geqslant\left(2 p \mu_{0}-C\right)\|s\|_{L^{2}}^{2}, \quad \text { for } s \in \Omega^{\neq q}\left(X, L^{p} \otimes E\right) .
$$

Proof. By (1.4) and (1.8), for $s \in \Omega^{0, \bullet}\left(X, L^{p} \otimes E\right)$,

$$
\left\|D_{p} s\right\|_{L^{2}}^{2}=\left\{\left\|\nabla^{\Lambda^{0, \bullet} \otimes L^{p} \otimes E} s\right\|_{L^{2}}^{2}-p\langle\tau(x) s, s\rangle\right\}-2 p\left\langle\omega_{d} s, s\right\rangle+\left\langle\left(\frac{1}{4} r^{X}+\mathbf{c}(R)\right) s, s\right\rangle .
$$

We consider now $s \in \mathscr{C}^{\infty}\left(X, L^{p} \otimes E^{\prime}\right)$, where $E^{\prime}=E \otimes \Lambda\left(T^{*(0,1)} X\right)$. By [23, Corollary 2.4] which is a direct consequence of the Lichnerowicz formula (cf. also [20, Theorem 1], [10, Theorem 2.1], [13, Theorem 4.4]), there exists $C>0$ such that for any $p>0, s \in \mathscr{C}^{\infty}\left(X, L^{p} \otimes E^{\prime}\right)$, we have

$$
\left\|\nabla^{L^{p} \otimes E^{\prime}} s\right\|_{L^{2}}^{2}-p\langle\tau(x) s, s\rangle \geqslant-C\|s\|_{L^{2}}^{2} .
$$

If $s \in \Omega^{\neq q}\left(X, L^{p} \otimes E\right)$, the second term of (1.10), $-2 p\left\langle\omega_{d} s, s\right\rangle$ is bounded below by $2 p \mu_{0}\|s\|_{L^{2}}^{2}$, while the third term of (1.10) is $\mathscr{O}\left(\|s\|_{L^{2}}^{2}\right)$. The proof of (1.9) is completed.

Set

$$
\begin{aligned}
D_{p}^{+} & =\left.D_{p}\right|_{\Omega^{0, \text { even }}}, \quad D_{p}^{-}=\left.D_{p}\right|_{\Omega^{0, \mathrm{odd}}}, \\
o_{q} & =-\quad \text { if } q \text { is even; } \quad o_{q}=+ \text { if } q \text { is odd } .
\end{aligned}
$$

By using the trick of the proof of Mckean-Singer formula and Theorem 1.1, the same proof as in [23, §3] gives the following extension of [23, Theorem 1.1]. For any operator $A$, we denote by $\operatorname{Spec}(A)$ the spectrum of $A$.

Theorem 1.2. There exists $C>0$ such that for $p \in \mathbb{N}$,

$$
\operatorname{Spec}\left(D_{p}^{2}\right) \subset\{0\} \cup\left[2 p \mu_{0}-C,+\infty[.\right.
$$

For p large enough, we have

$$
\operatorname{Ker} D_{p}^{o_{q}}=\{0\} \text {. }
$$

1.3. Off-diagonal asymptotic expansion of Bergman kernel. The existence of the spectral gap expressed in Theorem 1.2 allows us to obtain immediately as in [15. Prop. 4.1] the far off-diagonal behavior of the Bergman kernel. Namely, for any $l, m \in \mathbb{N}$ and $\varepsilon>0$, there exists $C_{l, m, \varepsilon}>0$ such that for $p \geqslant 1, x, x^{\prime} \in X$, $d\left(x, x^{\prime}\right)>\varepsilon$,

$$
\left|P_{p}\left(x, x^{\prime}\right)\right|_{\mathscr{C}^{m}(X \times X)} \leqslant C_{l, m, \varepsilon} p^{-l} .
$$

We denote by $I_{\operatorname{det}\left(\bar{W}^{*}\right) \otimes E}$ the orthogonal projection from $\mathbf{E}:=\Lambda\left(T^{*(0,1)} X\right) \otimes E$ onto $\operatorname{det}\left(\bar{W}^{*}\right) \otimes E$. Let $\pi: T X \times_{X} T X \rightarrow X$ be the natural projection from the fiberwise 
product of $T X$ on $X$. Let $\nabla^{\operatorname{End}(\mathbf{E})}$ be the connection on $\operatorname{End}\left(\Lambda\left(T^{*(0,1)} X\right) \otimes E\right)$ induced by $\nabla^{\text {Cliff }}$ and $\nabla^{E}$.

For $x_{0} \in X$, we identify $L_{Z}, E_{Z}$ and $\left(E_{p}\right)_{Z}$ for $Z \in B^{T_{x_{0}} X}(0, \varepsilon)$ to $L_{x_{0}}, E_{x_{0}}$ and $\left(E_{p}\right)_{x_{0}}$ by parallel transport with respect to the connections $\nabla^{L}, \nabla^{E}$ and $\nabla^{E_{p}}$ along the curve $\gamma_{Z}:[0,1] \ni u \rightarrow \exp _{x_{0}}^{X}(u Z)$. Under this identification and (1.15), we will view $P_{p}\left(x, x^{\prime}\right)$ as a smooth section $P_{p, x_{0}}\left(Z, Z^{\prime}\right),\left(Z, Z^{\prime} \in B^{T_{x_{0}} X}(0, \varepsilon)\right)$, of $\pi^{*}\left(\operatorname{End}\left(\Lambda\left(T^{*(0,1)} X\right) \otimes\right.\right.$ $E))$ on $T X \times_{X} T X$. And $\nabla^{\operatorname{End}(\mathbf{E})}$ induces naturally a $\mathscr{C}^{m}$-norm for the parameter $x_{0} \in X$.

Let $d v_{T X}$ be the Riemannian volume form on $\left(T_{x_{0}} X, g^{T_{x_{0}} X}\right)$. Let $\kappa(Z)$ be the smooth positive function defined by the equation

$$
d v_{X}(Z)=\kappa(Z) d v_{T X}(Z),
$$

with $\kappa(0)=1$. We denote by $\operatorname{det}_{\mathbb{C}}$ for the determinant function on the complex bundle $T^{(1,0)} X$, and $\left|\mathbf{J}_{x_{0}}\right|=\left(-\mathbf{J}_{x_{0}}^{2}\right)^{1 / 2}$. Denote by $\nabla_{U}$ the ordinary differentiation operator on $T_{x_{0}} X$ in the direction $U$. On $T_{x_{0}} X \simeq \mathbb{R}^{2 n}$, set

$$
L_{2, \mathbb{C}}^{0}=-\sum_{j}\left(\nabla_{e_{j}}+\frac{1}{2} R_{x_{0}}^{L}\left(Z, e_{j}\right)\right)^{2}-\tau_{x_{0}} .
$$

Let $P\left(Z, Z^{\prime}\right)$ be the Bergman kernel of $L_{2, \mathbb{C}}^{0}$, i.e. the smooth kernel of the orthogonal projection from $L^{2}\left(\mathbb{R}^{2 n}, \mathbb{C}\right)$ onto $\operatorname{Ker} L_{2, \mathbb{C}}^{0}$. Then for $Z, Z^{\prime} \in T_{x_{0}} X$,

$$
P\left(Z, Z^{\prime}\right)=\operatorname{det}_{\mathbb{C}}\left(\left|\mathbf{J}_{x_{0}}\right|\right) \exp \left(-\frac{\pi}{2}\left\langle\left|\mathbf{J}_{x_{0}}\right|\left(Z-Z^{\prime}\right),\left(Z-Z^{\prime}\right)\right\rangle-\pi \sqrt{-1}\left\langle\mathbf{J}_{x_{0}} Z, Z^{\prime}\right\rangle\right) \text {. }
$$

The main result of this part is the following extension of [15, Theorem 4.18'].

Theorem 1.3. There exist polynomials $J_{r}\left(Z, Z^{\prime}\right) \in \operatorname{End}\left(\Lambda\left(T^{*(0,1)} X\right) \otimes E\right)_{x_{0}}\left(x_{0} \in X\right)$, in $Z, Z^{\prime}$ with the same parity as $r$ and with $\operatorname{deg} J_{r} \leqslant 3 r$, whose coefficients are polynomials in $R^{T X}, R^{T^{(1,0)} X}, R^{E}$ (and $R^{L}$ ) and their derivatives of order $\leqslant 2 r-1$ (resp. $\leqslant 2 r$ ) and reciprocals of linear combinations of eigenvalues of $\mathbf{J}$ at $x_{0}$, such that by setting

$$
P_{x_{0}}^{(r)}\left(Z, Z^{\prime}\right)=J_{r}\left(Z, Z^{\prime}\right) P\left(Z, Z^{\prime}\right), \quad J_{0}\left(Z, Z^{\prime}\right)=\operatorname{det}_{\mathbb{C}}\left(\left|\mathbf{J}_{x_{0}}\right|\right) I_{\operatorname{det}\left(\bar{W}^{*}\right) \otimes E},
$$

the following statement holds: There exist $C^{\prime \prime}>0$ such that for any $k, m, m^{\prime} \in \mathbb{N}$, there exist $N \in \mathbb{N}$ and $C>0$ with

$$
\begin{aligned}
& \left|\frac{\partial^{|\alpha|+\left|\alpha^{\prime}\right|}}{\partial Z^{\alpha} \partial Z^{\prime \alpha^{\prime}}}\left(\frac{1}{p^{n}} P_{p}\left(Z, Z^{\prime}\right)-\sum_{r=0}^{k} P^{(r)}\left(\sqrt{p} Z, \sqrt{p} Z^{\prime}\right) \kappa^{-1}\left(Z^{\prime}\right) p^{-r / 2}\right)\right|_{\mathscr{C}^{m^{\prime}}(X)} \\
& \leqslant C p^{-(k+1-m) / 2}\left(1+|\sqrt{p} Z|+\left|\sqrt{p} Z^{\prime}\right|\right)^{N} \exp \left(-\sqrt{C^{\prime \prime} \mu_{0}} \sqrt{p}\left|Z-Z^{\prime}\right|\right)+\mathscr{O}\left(p^{-\infty}\right) .
\end{aligned}
$$

for any $\alpha, \alpha^{\prime} \in \mathbb{N}^{n}$, with $|\alpha|+\left|\alpha^{\prime}\right| \leqslant m$, any $Z, Z^{\prime} \in T_{x_{0}} X$ with $|Z|,\left|Z^{\prime}\right| \leqslant \varepsilon$ and any $x_{0} \in X, p \geqslant 1$.

Here $\mathscr{C}^{m^{\prime}}(X)$ is the $\mathscr{C}^{m^{\prime}}$-norm for the parameter $x_{0} \in X$. We say that a term $T=\mathscr{O}\left(p^{-\infty}\right)$ if for any $l, l_{1} \in \mathbb{N}$, there exists $C_{l, l_{1}}>0$ such that the $\mathscr{C}^{l_{1}}$-norm of $T$ is dominated by $C_{l, l_{1}} p^{-l}$. 
Proof. By using Theorems 1.1, 1.2, we know the arguments in [15, §4.1-4.3] go through without any change. By (1.4), (1.8), as in [15, (4.105)], the corresponding limit operator here is still

$$
L_{2}^{0}=L_{2, \mathbb{C}}^{0}-2 \omega_{d, x_{0}} .
$$

Let $e^{-u L_{2, \mathbb{C}}^{0}}\left(Z, Z^{\prime}\right), e^{-u L_{2}^{0}}\left(Z, Z^{\prime}\right)$ be the smooth kernels of $e^{-u L_{2, \mathrm{C}}^{0}}, e^{-u L_{2}^{0}}$ with respect to $d v_{T X}\left(Z^{\prime}\right)$. Now from (1.21) (cf. [8, (6.37), (6.38)]), we need to replace [15, (4.106)] by the following equations

$$
\begin{aligned}
& e^{-u L_{2, \mathbb{C}}^{0}}\left(Z, Z^{\prime}\right)=\operatorname{det}_{\mathbb{C}}\left(\frac{\left|\mathbf{J}_{x_{0}}\right|}{1-e^{-4 \pi u\left|\mathbf{J}_{x_{0}}\right|}}\right) \exp \left(-\frac{1}{2}\left\langle\frac{\pi\left|\mathbf{J}_{x_{0}}\right|}{\tanh \left(2 \pi u\left|\mathbf{J}_{x_{0}}\right|\right)} Z, Z\right\rangle\right. \\
& \left.-\frac{1}{2}\left\langle\frac{\pi\left|\mathbf{J}_{x_{0}}\right|}{\tanh \left(2 \pi u\left|\mathbf{J}_{x_{0}}\right|\right)} Z^{\prime}, Z^{\prime}\right\rangle+\left\langle\frac{\pi\left|\mathbf{J}_{x_{0}}\right|}{\sinh \left(2 \pi u\left|\mathbf{J}_{x_{0}}\right|\right)} e^{-2 \pi \sqrt{-1} u \mathbf{J}_{x_{0}}} Z, Z^{\prime}\right\rangle\right), \\
& e^{-u L_{2}^{0}}\left(Z, Z^{\prime}\right)=e^{-u L_{2, \mathbb{C}}^{0}}\left(Z, Z^{\prime}\right) e^{2 u \omega_{d, x_{0}}} .
\end{aligned}
$$

Observe that for $\omega_{d, x_{0}} \in \operatorname{End}\left(\Lambda\left(T^{*(0,1)} X\right)\right)_{x_{0}}$, by (1.7),

$$
\begin{aligned}
& \text { Ker } \omega_{d, x_{0}}=\operatorname{det}\left(\bar{W}^{*}\right)_{x_{0}}, \\
& \omega_{d, x_{0}} \leqslant-\mu_{0} \quad \text { on }\left(\operatorname{det}\left(\bar{W}^{*}\right)_{x_{0}}\right)^{\perp}=\text { the orthogonal complement of } \operatorname{det}\left(\bar{W}^{*}\right) .
\end{aligned}
$$

Thus from [15, §4.4, 4.5], we get Theorem 1.3.

If we take $Z, Z^{\prime}=0$ in Theorem 1.3 we infer the following result by the same argument in [15, §4.5].

Theorem 1.4. There exist smooth coefficients $\boldsymbol{b}_{r}(x) \in \operatorname{End}\left(\Lambda\left(T^{*(0,1)} X\right) \otimes E\right)_{x}$ which are polynomials in $R^{T X}, R^{T^{(1,0)} X}, R^{E}$ (and $R^{L}$ ) and their derivatives of order $\leqslant 2 r-1$ (resp. $\leqslant 2 r$ ) and reciprocals of linear combinations of eigenvalues of $\mathbf{J}$ at $x$, such that

$$
\boldsymbol{b}_{0}=\operatorname{det}_{\mathbb{C}}(|\mathbf{J}|) I_{\operatorname{det}\left(\bar{W}^{*}\right) \otimes E}
$$

and for any $k, l \in \mathbb{N}$, there exists $C_{k, l}>0$ with

$$
\left|P_{p}(x, x)-\sum_{r=0}^{k} \boldsymbol{b}_{r}(x) p^{n-r}\right|_{\mathscr{C} l} \leqslant C_{k, l} p^{n-k-1} .
$$

for any $x \in X$ and $p \in \mathbb{N}$. Moreover, the expansion is uniform in that for any $k, l \in \mathbb{N}$, there is an integer $s$ such that if all data $\left(g^{T X}, h^{L}, \nabla^{L}, h^{E}, \nabla^{E}\right)$ run over a set which are bounded in $\mathscr{C}^{s}$ and with $g^{T X}$ bounded below, there exists the constant $C_{k, l}$ independent of $g^{T X}$, and the $\mathscr{C}^{l}$-norm in (1.25) includes also the derivatives on the parameters.

1.4. Holomorphic case revisited. In this Section, we suppose that $(X, J)$ is a complex manifold with complex structure $J$ and $E, L$ are holomorphic vector bundles on $X$. We assume that $\nabla^{E}, \nabla^{L}$ are the holomorphic Hermitian (i.e. Chern) connections on $\left(E, h^{E}\right),\left(L, h^{L}\right)$ and moreover, $\omega:=\frac{\sqrt{-1}}{2 \pi} R^{L}$ defines a symplectic form on $X$. Therefore the signature of the curvature $\frac{\sqrt{-1}}{2 \pi} R^{L}$ (i.e. number of negative 
and positive eigenvalues) with respect to any Riemannian metric compatible with $J$ will be the same. Let $g^{T X}$ be any Riemannian metric on $T X$ compatible with $J$. Since $g^{T X}$ is not necessarily Kähler, $\mathbf{J} \neq J$ in (1.5) in general. Recall the number $q$ is defined by (1.6), i.e. is the number of negative eigenvalues of $\omega$. Set

$$
\Theta(X, Y)=g^{T X}(J X, Y) .
$$

Then the 2-form $\Theta$ need not be closed (the convention here is different to [6, (2.1)] by a factor -1 ).

Let $\bar{\partial}^{L^{p} \otimes E, *}$ be the formal adjoint of the Dolbeault operator $\bar{\partial}^{L^{p} \otimes E}$ on the Dolbeault complex $\Omega^{0, \bullet}\left(X, L^{p} \otimes E\right)$ with the scalar product induced by $g^{T X}, h^{L}, h^{E}$ as in (1.2). Set

$$
D_{p}=\sqrt{2}\left(\bar{\partial}^{L^{p} \otimes E}+\bar{\partial}^{L^{p} \otimes E, *}\right) .
$$

We denote by $\square^{L^{p} \otimes E}=\bar{\partial}^{L^{p} \otimes E} \bar{\partial}^{L^{p} \otimes E, *}+\bar{\partial}^{L^{p} \otimes E, *} \bar{\partial}^{L^{p} \otimes E}$ the Kodaira-Laplacian. Then $D_{p}^{2}=2 \square^{L^{p} \otimes E}$ it is twice the Kodaira-Laplacian and preserves the $\mathbb{Z}$-grading of $\Omega^{0, \bullet}\left(X, L^{p} \otimes E\right)$. By Hodge theory, we know that for any $k, p \in \mathbb{N}$,

$$
\left.\operatorname{Ker} D_{p}\right|_{\Omega^{0, k}}=\left.\operatorname{Ker} D_{p}^{2}\right|_{\Omega^{0, k}} \simeq H^{0, k}\left(X, L^{p} \otimes E\right),
$$

where $H^{0, \bullet}\left(X, L^{p} \otimes E\right)$ is the Dolbeault cohomology. Here $D_{p}$ is not a $\operatorname{spin}^{c}$ Dirac operator on $\Omega^{0, \bullet}\left(X, L^{p} \otimes E\right)$, and $D_{p}^{2}$ is not a renormalized Bochner-Laplacian as in [24], so we cannot apply directly Theorems 1.1] and 1.2. Now we explain how to recover the conclusions of these theorems in the case of $D_{p}$. The first step is to exhibit the spectral gap.

Theorem 1.5. The statements of Theorems 1.1] and 1.2 still hold for the operator $D_{p}$ defined by (1.27). In particular, for p large enough,

$$
H^{0, k}\left(X, L^{p} \otimes E\right)=0 \text { for } k \neq q .
$$

Proof. As we will use [7, Theorem 2.3] to study the Bergman kernel in the sequel, we prove Theorem 1.5] by explaining [7, Theorem 2.3].

Let $S^{-B}$ denote the 1 -form with values in the antisymmetric elements of $\operatorname{End}(T X)$ which satisfies

$$
\left\langle S^{-B}(U) V, W\right\rangle=-\frac{\sqrt{-1}}{2}((\partial-\bar{\partial}) \Theta)(U, V, W), \quad \text { for } U, V, W \in T X .
$$

The Bismut connection $\nabla^{-B}$ on $T X$ is defined by

$$
\nabla^{-B}=\nabla^{T X}+S^{-B} \text {. }
$$

Then by [7, Prop. 2.5], $\nabla^{-B}$ preserves the metric $g^{T X}$ and the complex structure of $T X$. Let $\nabla^{\text {det }}$ be the holomorphic Hermitian connection on $\operatorname{det}\left(T^{(1,0)} X\right)$ whose curvature is denoted $R^{\text {det }}$. Then these two connections induce naturally an unique connection on $\Lambda\left(T^{*(0,1)} X\right)$ which preserves its $\mathbb{Z}$-grading, and with the connections $\nabla^{L}, \nabla^{E}$, we get a connection $\nabla^{-B, E_{p}}$ on $\Lambda\left(T^{*(0,1)} X\right) \otimes L^{p} \otimes E$. Let $\left(\nabla^{-B, E_{p}}\right)^{*}$ be the formal adjoint of $\nabla^{-B, E_{p}}$. Let $C(T X)$ be the Clifford bundle of $T X$. We define a map ${ }^{c}: \Lambda\left(T^{*} X\right) \rightarrow C(T X)$, by sending $e^{i_{1}} \wedge \cdots \wedge e^{i_{j}}$ to $c\left(e_{i_{1}}\right) \cdots c\left(e_{i_{j}}\right)$ for $i_{1}<\cdots<i_{j}$. For 
$B \in \Lambda^{3}\left(T^{*} X\right)$, set $|B|^{2}=\sum_{i<j<k}\left|B\left(e_{i}, e_{j}, e_{k}\right)\right|^{2}$. Then we can formulate [7, Theorem 2.3] as following:

$$
\begin{aligned}
D_{p}^{2}=\left(\nabla^{-B, E_{p}}\right)^{*} \nabla^{-B, E_{p}}+\frac{r^{X}}{4}+{ }^{c}\left(R^{E}+p R^{L}+\right. & \left.\frac{1}{2} R^{\mathrm{det}}\right) \\
& +\frac{\sqrt{-1}}{2} c(\bar{\partial} \partial \Theta)-\frac{1}{8}|(\partial-\bar{\partial}) \Theta|^{2} .
\end{aligned}
$$

(1.32) can be seen as a Bochner-Kodaira-Nakano type formula.) By using (1.8), (1.11) and (1.32), as in Theorems 1.1 and 1.2, we see that the conclusions of these theorems still hold for the operator $D_{p}$ defined in (1.27). In particular (1.9) holds. Now from (1.9) and (1.28), we get (1.29).

Remark 1.6. The vanishing result (1.29) is Andreotti-Grauert's coarse vanishing theorem [1, §23] (where it is proved by using the cohomology finiteness theorem for the disc bundle of $L^{*}$ ). It can also be deduced, as shown by Griffiths [19, p. 432], from the usual Bochner-Kodaira-Nakano formula [16, 19, 26]. The latter implies the following inequality for $u \in \Omega^{m, k}\left(X, L^{p} \otimes E\right)$ :

$$
\begin{aligned}
\frac{3}{2}\left(\left\|\bar{\partial}^{L^{p} \otimes E} u\right\|^{2}+\left\|\bar{\partial}^{L^{p} \otimes E, *} u\right\|^{2}\right) \geqslant \int_{X} & \left\langle\left[\sqrt{-1}\left(p R^{L}+R^{E}\right), \Lambda\right] u, u\right\rangle d v_{X} \\
& -\frac{1}{2}\left(\|T u\|^{2}+\left\|T^{*} u\right\|^{2}+\|\bar{T} u\|^{2}+\left\|\bar{T}^{*} u\right\|^{2}\right)
\end{aligned}
$$

where $\Lambda=i(\Theta)$ denotes the interior product with $\Theta$ and $T=[\Lambda, \partial \Theta]$ is the torsion of the metric $g^{T X}$. We have pointwise

$$
\left\langle\left[\sqrt{-1} R^{L}, \Lambda\right] u, u\right\rangle \geqslant\left(\lambda_{1}+\ldots+\lambda_{k}-\lambda_{n-m+1}-\ldots-\lambda_{n}\right)|u|^{2},
$$

where $\lambda_{1} \leqslant \lambda_{2} \leqslant \ldots \leqslant \lambda_{n}$ are the eigenvalues of $\sqrt{-1} R^{L}$ with respect to $\Theta$. If $m=0$ the right-hand side becomes $\left(-\lambda_{k+1}-\ldots-\lambda_{n}\right)|u|^{2}$. As in [26, Lemma 4.3] we can restrict ourselves to those metrics $g^{T X}$ such that the negative eigenvalues $\lambda_{1}, \ldots, \lambda_{q}$ are very large and the positive ones $\lambda_{q+1}, \ldots, \lambda_{n}$ are very small in absolute value. Therefore, for $k<q$ there exists a constant $\mu_{1}>0$ such that $-\lambda_{k+1}-\ldots-\lambda_{n} \geqslant \mu_{1}$ on $X$. By (1.33) we obtain (1.9) for $u \in \Omega^{0,<q}\left(X, L^{p} \otimes E\right)=\oplus_{k<q} \Omega^{0, k}\left(X, L^{p} \otimes E\right)$.

In order to consider the case $k>q$, we apply again (1.33) and (1.34) for $(n, q)$ $L^{p} \otimes K_{X}^{*} \otimes E$-valued forms, which involves another change of metric, for which $\lambda_{1}, \ldots, \lambda_{q}$ are small and $\lambda_{q+1}, \ldots, \lambda_{n}$ are large in absolute value. Thus we get (1.9) also for $u \in \Omega^{0,>q}\left(X, L^{p} \otimes E\right)=\oplus_{k>q} \Omega^{0, k}\left(X, L^{p} \otimes E\right)$, but for yet another class of metrics $g^{T X}$. Of course, the estimates just obtained entail immediately (1.29).

We see however that by using (1.33) the essential estimate (1.9) for a fixed metric $g^{T X}$ seems out of reach, as well as the existence of the spectral gap (1.13).

By Theorem 1.5 the kernel of $D_{p}^{2}$ is concentrated in degree $q$. We consider thus the Bergman kernel of $D_{p}^{2}$ in this particular degree. Let $P_{p}^{0, q}\left(x, x^{\prime}\right)$ be the smooth kernel with respect to $d v_{X}\left(x^{\prime}\right)$ of the orthogonal projection from $\Omega^{0, q}\left(X, L^{p} \otimes E\right)$ on $\operatorname{Ker} D_{p}^{2}$. 
Theorem 1.7. The Bergman kernel $P_{p}^{0, q}\left(x, x^{\prime}\right)$ has a full off-diagonal asymptotic expansion analogous to (1.20) with $J_{0}=\operatorname{det}_{\mathbb{C}}(|\mathbf{J}|) I_{\operatorname{det}\left(\bar{W}^{*}\right) \otimes E}$ as $p \rightarrow \infty$.

Proof. We use now the connection $\nabla^{-B, E_{p}}$ instead of $\nabla^{E_{p}}$ in [15, §3]. Then by (1.9) and (1.32), everything goes through perfectly well and as in [15, Theorem 4.18], so we can directly apply the result from [15] to get the full off-diagonal asymptotic expansion of the Bergman kernel. As the above construction preserves the $\mathbb{Z}$ grading on $\Omega^{0, \bullet}\left(X, L^{p} \otimes E\right)$, we can directly work on $\Omega^{0, q}\left(X, L^{p} \otimes E\right)$.

Remark 1.8. From the arguments here and [23], [24, §3.5], we get naturally the covering version of Sections 1.2]1.4

1.5. Relation to the tangential Cauchy-Riemann complex. If $q=0$, i.e. J has only positive eigenvalues, Theorem 1.7 boils down to [24, Theorem 3.9]. Theorem 1.7 for $x=x^{\prime}$ is first due to Zelditch [28] and Catlin [14] and is based on the Boutet de Monvel-Sjöstrand parametrix [12] for the Szegö projector on CR functions on the boundary of the "Grauert tube" associated to $L$. For general $q \neq 0$, Berman and Sjöstrand [5] recently studied the asymptotic expansion $P_{p}\left(x, x^{\prime}\right)$, too. They use an approach of Melin-Sjöstrand originating in the theory of Fourier integral operators with complex phase.

In this section we briefly discuss the link between our analysis for $q \neq 0$ and the the kernel of the Szegö projector on $(0, k)$ forms on the boundary of the Grauert tube. We use the notations and assumptions from Section 1.4.

Let $Y=\left\{u \in L^{*},|u|_{h^{L^{*}}}=1\right\}$ be the unit circle bundle in $L^{*}$. $Y$ is a real hypersurface in the complex manifold $L^{*}$ which the boundary of the disc bundle $D=\left\{u \in L^{*},|u|_{h^{L^{*}}}<1\right\}$, with defining function $\varrho=|u|_{h^{L^{*}}}-1$. The Levi form of $\varrho$ restricted to the complex tangent plane of $Y$ coincides with the pull-back of $\omega$ through the canonical projection $\pi: Y \rightarrow X$. Hence it has $q$ negative and $n-q$ positive eigenvalues. We denote by $T^{*(0,1)}(Y)=T^{*(0,1)} L^{*} \cap\left(T^{*} Y \otimes_{\mathbb{R}} \mathbb{C}\right)$ the bundle of $(0,1)$-forms tangential to $Y$ and by $\Omega^{0, k}(Y)$ the space of smooth sections of $\Lambda^{k}\left(T^{*(0,1)} Y\right)$. The $\bar{\partial}$ operator on the ambient manifold $L^{*}$ induces as usual a tangential Cauchy-Riemann complex on the hypersurface $Y$ [21, 2, 3].

$$
0 \longrightarrow \Omega^{0,0}(Y) \stackrel{\bar{\partial}_{b}}{\longrightarrow} \Omega^{0,1}(Y) \stackrel{\bar{\partial}_{b}}{\longrightarrow} \cdots \stackrel{\bar{\partial}_{b}}{\longrightarrow} \Omega^{0, n}(Y) \longrightarrow 0 .
$$

The $\bar{\partial}_{b}$ operator commutes with the action of $S^{1}$ on $Y$.

The connection $\nabla^{L}$ on $L$ induces a connection on the $S^{1}$-principal bundle $\pi: Y \rightarrow$ $X$, and let $T^{H} Y \subset T Y$ be the corresponding horizontal bundle. Let us introduce the Riemannian metric $g^{T Y}=\pi^{*}\left(g^{T X}\right) \oplus d \vartheta^{2}$ on $T Y=T^{H} Y \oplus T S^{1}$. We will denote by $\bar{\partial}_{b}^{*}$ the formal adjoint of $\bar{\partial}_{b}$ with respect to this metric and form the Kohn-Laplacian

$$
\square_{b}=\bar{\partial}_{b} \bar{\partial}_{b}^{*}+\bar{\partial}_{b}^{*} \bar{\partial}_{b} .
$$

The operators $\bar{\partial}_{b}^{*}$ and $\square_{b}$ also commute with the action of $S^{1}$ on $Y$. Consider the space $\mathscr{C}^{\infty}(Y)_{p}$ of smooth functions $f$ on $Y$ which transform under the action 
$(y, \vartheta) \mapsto e^{i \vartheta} y$ of $S^{1}$ according to the law

$$
f\left(e^{i \vartheta} y\right)=e^{i p \vartheta} f(y) .
$$

This space of functions can be identified naturally with the space of smooth sections $\Omega^{0,0}\left(X, L^{p}\right)$. More generally, the space of sections $\Omega^{0, k}(Y)_{p}$ which transform under the action of $S^{1}$ according to the law (1.37) can be naturally identified with the space $\Omega^{0, k}\left(X, L^{p}\right)$. Therefore, for each integer $p$, we get a subcomplex $\left(\Omega^{0} \bullet(Y)_{p}, \bar{\partial}_{b}\right)$ of the tangential Cauchy-Riemann complex (1.35), isomorphic to the Dolbeault complex $\left(\Omega^{0, \bullet}\left(X, L^{p}\right), \bar{\partial}^{L^{p}}\right)$. Moreover, the action of $\square_{b}$ on $\Omega^{0, \bullet}(Y)_{p}$ is identical to the action of the Kodaira-Laplacian $\square^{L^{p}}$ on $\Omega^{0} \cdot \bullet\left(X, L^{p}\right)$, via the the complex isomorphism just mentioned.

Let us consider the spaces of $\square_{b}$-harmonic spaces

$$
\mathscr{H}^{0, k}(Y)=\left.\operatorname{ker} \square_{b}\right|_{\Omega^{0, k}(Y)}, \quad \mathscr{H}^{0, k}(Y)_{p}=\left.\operatorname{ker} \square_{b}\right|_{\Omega^{0, k}(Y)_{p}} .
$$

Then

$$
\mathscr{H}^{0, k}(Y)=\oplus_{p \in \mathbb{Z}} \mathscr{H}^{0, k}(Y)_{p} \cong \oplus_{p \in \mathbb{Z}} H^{0, k}\left(X, L^{p}\right)
$$

where $H^{0, k}\left(X, L^{p}\right):=\left.\operatorname{ker} \square^{L^{p}}\right|_{\Omega^{0, k}\left(X, L^{p}\right)}$ is the space of harmonic forms (1.28).

The Szegö projector $\Pi^{0, k}$ is the orthogonal projection from $\Omega^{0, k}(Y)$ to $\mathscr{H}^{0, k}(Y)$. The Szegö projector $\Pi^{0, k}$ is finite dimensional for the degrees $k \neq q, n-q$. This follows from the decomposition (1.39) and the vanishing theorem of AndreottiGrauert (1.29) applied for both $L$ and $L^{*}$. It shows that there exits $p_{0} \in \mathbb{N}$ such that $H^{0, k}\left(X, L^{p}\right)=0$ for all $p \in \mathbb{Z}$ with $|p| \geqslant p_{0}$ and all $k \neq q, n-q$ (note that $R^{L^{*}}=-R^{L}$ has $n-q$ negative and $q$ positive eigenvalues).

On the other hand, the Szegö projector $\Pi^{0, k}$ is infinite dimensional for the degrees $k=q, n-q$ as shown by (1.29) combined with the Riemann-Roch-Hirzebruch formula, which in turn is a consequence of the integration of the asymptotic expansion from Theorem 1.7 over the manifold $X$. To obtain the result for $k=n-q$ we have to replace $L$ by $L^{*}$ in the above mentioned results.

The description of the dimension of the harmonic spaces is consistent with the general geometric information from [2, 3] and [11, p. 626], where general hypersurfaces $Y$ are considered, with non-degenerate Levi form of signature $(q, n-q)$.

The relation between the Bergman kernels $P_{p}^{0, q}$ considered in Theorem 1.7 and the Szegö kernel $\Pi^{0, q}$ is given by

$$
P_{p}^{0, q}(x, x)=\frac{1}{2 \pi} \int_{S^{1}} \Pi^{0, q}\left(e^{i \vartheta} y, y\right) e^{-i p \vartheta} d \vartheta
$$

where $x \in X$ and $y \in Y$ satisfy $\pi(y)=x$. This means that the $P_{p}^{0, q}(x, x)$ represent the Fourier coefficients of the distribution $\Pi^{0, q}(y, y)$. Since we know the asymptotic expansion of $P_{p}^{0, q}(x, x)$ as $p \rightarrow \infty$ given in Theorem 1.7 we can recover from (1.40) the restriction on the diagonal of the Szegö kernel $\Pi^{0, q}$.

It could also be possible to work in the opposite direction and start with the Szegö kernel. Namely, using a similar analysis as the one of Boutet de Monvel and Sjöstrand [12] one can find the parametrix of the Szegö kernel $\Pi^{0, q}$ and determine 
its singularity on the diagonal. Then, working as Zelditch [28] (where the case $q=0$ is considered) one can deduce the asymptotic of $P_{p}^{0, q}(x, x)$ for $p \rightarrow \infty$.

The same discussion applies to $\Pi^{0, n-q}$ and the Bergman kernels $P_{p}^{0, n-q}$ associated to $L^{*}$.

\section{THE COEFFICIENT $\boldsymbol{b}_{1}$}

This Section is organized as follows. In Section 2.1, we state our main result, the formula for the coefficient $b_{1}$ for the $\operatorname{spin}^{c}$ Dirac operator. In Section 2.2, we obtain an asymptotic expansion of the rescaled $\operatorname{spin}^{c}$ Dirac operator $L_{2}^{t}$ (cf. (2.4)) in normal coordinates. In Section 2.3, we finally compute the coefficient $\boldsymbol{b}_{1}$.

2.1. Main result. We use the notation in Section 1.1. We denote by $I_{\mathbb{C} \otimes E}$ the projection from $\Lambda\left(T^{*(0,1)} X\right) \otimes E$ onto $\mathbb{C} \otimes E$ under the decomposition $\Lambda\left(T^{*(0,1)} X\right)=\mathbb{C} \oplus$ $\Lambda^{>0}\left(T^{*(0,1)} X\right)$. For any tensor $\psi$ on $X$, we denote by $\nabla^{X} \psi$ the covariant derivative of $\psi$ induced by $\nabla^{T X}$. Thus $\nabla^{X} J \in T^{*} X \otimes \operatorname{End}(T X), \nabla^{X} \nabla^{X} J \in T^{*} X \otimes T^{*} X \otimes \operatorname{End}(T X)$. Let $\left\{w_{j}\right\}$ be an orthonormal basis of $\left(T^{(1,0)} X, g^{T X}\right)$, and its dual basis $\left\{w^{j}\right\}$. Let $\left\{e_{i}\right\}_{1 \leqslant i \leqslant 2 n}$ be an orthonormal basis of $\left(T X, g^{T X}\right)$. Then we denote by $\left|\nabla^{X} J\right|^{2}:=$ $\sum_{i j}\left|\left(\nabla_{e_{i}}^{X} J\right) e_{j}\right|^{2}$.

The following result is the main result of this paper.

Theorem 2.1. If $\mathbf{J}=J$, then for $b_{1}$ in (1.25), we have

$$
\begin{aligned}
\boldsymbol{b}_{1}(x) & =\frac{1}{8 \pi}\left[r^{X}+\frac{1}{4}\left|\nabla^{X} J\right|^{2}+4 R^{E}\left(w_{j}, \bar{w}_{j}\right)\right] I_{\mathbb{C} \otimes E}-\frac{1}{144 \pi} \sum_{k l}\left|\left(\nabla_{w_{k}}^{X} J\right) w_{l}\right|^{2} I_{\mathbb{C} \otimes E} \\
& +\frac{1}{288 \pi}\left\langle\left(\nabla_{\bar{w}_{k}}^{X} J\right) \bar{w}_{l}, \bar{w}_{m}\right\rangle\left\langle\left(\nabla_{w_{k}}^{X} J\right) w_{i}, w_{j}\right\rangle \bar{w}^{l} \wedge \bar{w}^{m} I_{\mathbb{C} \otimes E} i_{\bar{w}_{j}} \wedge i_{\bar{w}_{i}} \\
& -\frac{1}{8 \pi}\left(\frac{1}{3}\left\langle R^{T X} w_{i}, \bar{w}_{i}\right\rangle+R^{E}\right)\left(\bar{w}_{l}, \bar{w}_{m}\right) \bar{w}^{l} \wedge \bar{w}^{m} I_{\mathbb{C} \otimes E} \\
& +\frac{1}{8 \pi}\left(\frac{1}{3}\left\langle R^{T X} w_{i}, \bar{w}_{i}\right\rangle+R^{E}\right)\left(w_{l}, w_{m}\right) I_{\mathbb{C} \otimes E} i_{\bar{w}_{m}} \wedge i_{\bar{w}_{l}} .
\end{aligned}
$$

Especially,

$$
\left.\operatorname{Tr}\right|_{\Lambda\left(T^{*(0,1)} X\right)}\left[\boldsymbol{b}_{1}(x)\right]=\frac{1}{8 \pi}\left[r^{X}+\frac{1}{4}\left|\nabla^{X} J\right|^{2}+4 R^{E}\left(w_{j}, \bar{w}_{j}\right)\right] .
$$

The term $r^{X}+\frac{1}{4}\left|\nabla^{X} J\right|^{2}$ in (2.2) is called the Hermitian scalar curvature in the literature [17], [18, Chap. 10], [24] and is a natural substitute for the Riemannian scalar curvature in the almost-Kähler case. It was used by Donaldson [17] to define the moment map on the space of compatible almost-complex structures.

2.2. Taylor expansion of the operator $L_{2}^{t}$. To compare with [24, §1.2], in this part, we assume that $\omega(\cdot, J \cdot)$ is positive, i.e. $q=0$ in (1.6).

We fix $x_{0} \in X$. From now on, we identify $B^{T_{x_{0}} X}(0, \varepsilon)$ with $B^{X}\left(x_{0}, \varepsilon\right)$ by the exponential map $T_{x_{0}} X \ni Z \rightarrow \exp _{x_{0}}^{X}(Z) \in X$. We identify $L_{Z}, E_{Z}$ and $\left(E_{p}\right)_{Z}$ for $Z \in B^{T_{x_{0}} X}(0, \varepsilon)$ to $L_{x_{0}}, E_{x_{0}}$ and $\left(E_{p}\right)_{x_{0}}$ by parallel transport with respect to the connections $\nabla^{L}, \nabla^{E}$ and $\nabla^{E_{p}}$ along the curve $\gamma_{Z}:[0,1] \ni u \rightarrow u Z$. Let $\left\{e_{i}\right\}_{i}$ be an 
oriented orthonormal basis of $T_{x_{0}} X$. We also denote by $\left\{e^{i}\right\}_{i}$ the dual basis of $\left\{e_{i}\right\}$. Let $\widetilde{e}_{i}(Z)$ be the parallel transport of $e_{i}$ with respect to $\nabla^{T X}$ along the above curve.

Let $S_{L}$ be an unit vector of $L_{x_{0}}$. Using $S_{L}$ and the above discussion, we get an isometry $E_{p} \simeq\left(\Lambda\left(T^{*(0,1)} X\right) \otimes E\right)_{x_{0}}=: \mathbf{E}_{x_{0}}$ on $B^{T_{x_{0}} X}(0, \varepsilon)$. Under our identification, $h^{E_{p}}$ is $h^{\mathbf{E}_{x_{0}}}$ on $B^{T_{x_{0}} X}(0, \varepsilon)$.

For $s \in \mathscr{C}^{\infty}\left(T_{x_{0}} X, \mathbf{E}_{x_{0}}\right)$, set

$$
\|s\|_{0,0}^{2}=\int_{\mathbb{R}^{2 n}}|s(Z)|_{\left.h_{x_{0}}^{\Lambda\left(T^{*}(0,1)\right.} X\right) \otimes E}^{2} d v_{T X}(Z) .
$$

Denote by $\nabla_{U}$ the ordinary differentiation operator on $T_{x_{0}} X$ in the direction $U$. If $\alpha=\left(\alpha_{1}, \cdots, \alpha_{2 n}\right)$ is a multi-index, set $Z^{\alpha}=Z_{1}^{\alpha_{1}} \cdots Z_{2 n}^{\alpha_{2 n}}$. Let $\left(\partial^{\alpha} R^{L}\right)_{x_{0}}$ be the tensor $\left(\partial^{\alpha} R^{L}\right)_{x_{0}}\left(e_{i}, e_{j}\right)=\partial^{\alpha}\left(R^{L}\left(e_{i}, e_{j}\right)\right)_{x_{0}}$. We denote by $\mathcal{R}=\sum_{i} Z_{i} e_{i}=Z$ the radial vector field on $\mathbb{R}^{2 n}$. Recall that the function $\kappa$ was defined in (1.16). For $s \in \mathscr{C}^{\infty}\left(B^{T_{x_{0}} X}(0, \varepsilon), \mathbf{E}_{x_{0}}\right)$ and $Z \in B^{T_{x_{0}} X}(0, \varepsilon)$, for $t=\frac{1}{\sqrt{p}}$, set

$$
\begin{aligned}
& \left(S_{t} s\right)(Z)=s(Z / t), \quad \nabla_{t}=S_{t}^{-1} t \kappa^{\frac{1}{2}} \nabla^{E_{p}} \kappa^{-\frac{1}{2}} S_{t}, \\
& \nabla_{0, \cdot}=\nabla .+\frac{1}{2} R_{x_{0}}^{L}(\mathcal{R}, \cdot), \quad L_{2}^{t}=S_{t}^{-1} t^{2} \kappa^{\frac{1}{2}} D_{p}^{2} \kappa^{-\frac{1}{2}} S_{t} .
\end{aligned}
$$

By our trivialization, $L_{2}^{t}$ is self-adjoint with respect to $\|\cdot\|_{0,0}$ on $\mathscr{C}_{0}^{\infty}\left(B^{T_{x_{0}} X}(0, \varepsilon / t), \mathbf{E}_{x_{0}}\right)$. Note that comparing with [15, (4.37)], we conjugate with $\kappa^{1 / 2}$ in (2.4), which simplifies the computation of the coefficient $b_{1}$.

We adopt the convention that all tensors will be evaluated at the base point $x_{0} \in X$, and most of the time, we will omit the subscript $x_{0}$. Let $\mathscr{L}_{0}, \mathcal{O}_{1}, \mathcal{O}_{2}$ be the operators defined in [24, Theorem 1.4] associated the renormalized BochnerLaplacian $\Delta_{p, 0}$. Recall that $\tau=\sum_{j} R^{L}\left(w_{j}, \bar{w}_{j}\right), \omega_{d}=-R^{L}\left(w_{l}, \bar{w}_{m}\right) \bar{w}^{m} \wedge i_{\bar{w}_{l}}$. Thus we have

$$
\begin{aligned}
& \mathscr{L}_{0}=-\sum_{j}\left(\nabla_{0, e_{j}}\right)^{2}-\tau_{x_{0}}, \\
& \mathcal{O}_{1}(Z)=-\frac{2}{3}\left(\partial_{j} R^{L}\right)_{x_{0}}\left(\mathcal{R}, e_{i}\right) Z_{j} \nabla_{0, e_{i}}-\frac{1}{3}\left(\partial_{i} R^{L}\right)_{x_{0}}\left(\mathcal{R}, e_{i}\right)-\left(\partial_{\mathcal{R}} \tau\right)_{x_{0}}, \\
& \mathcal{O}_{2}(Z)=\frac{1}{3}\left\langle R_{x_{0}}^{T X}\left(\mathcal{R}, e_{i}\right) \mathcal{R}, e_{j}\right\rangle_{x_{0}} \nabla_{0, e_{i}} \nabla_{0, e_{j}} \\
& \quad+\left[\frac{2}{3}\left\langle R_{x_{0}}^{T X}\left(\mathcal{R}, e_{j}\right) e_{j}, e_{i}\right\rangle-\left(\frac{1}{2} \sum_{|\alpha|=2}\left(\partial^{\alpha} R^{L}\right)_{x_{0}} \frac{Z^{\alpha}}{\alpha !}+R_{x_{0}}^{E}\right)\left(\mathcal{R}, e_{i}\right)\right] \nabla_{0, e_{i}} \\
& -\frac{1}{4} \nabla_{e_{i}}\left(\sum_{|\alpha|=2}\left(\partial^{\alpha} R^{L}\right)_{x_{0}} \frac{Z^{\alpha}}{\alpha !}\left(\mathcal{R}, e_{i}\right)\right)-\frac{1}{9} \sum_{i}\left[\sum_{j}\left(\partial_{j} R^{L}\right)_{x_{0}}\left(\mathcal{R}, e_{i}\right) Z_{j}\right]^{2} \\
& -\frac{1}{12}\left[\mathscr{L}_{0},\left\langle R_{x_{0}}^{T X}\left(\mathcal{R}, e_{i}\right) \mathcal{R}, e_{i}\right\rangle_{x_{0}}\right]-\sum_{|\alpha|=2}\left(\partial^{\alpha} \tau\right)_{x_{0}} \frac{Z^{\alpha}}{\alpha !} .
\end{aligned}
$$


FIRST COEFFICIENTS OF THE ASYMPTOTIC EXPANSION OF THE BERGMAN KERNEL 13

Theorem 2.2. There are second order differential operators $L_{2}^{0}, \underline{\mathcal{Q}}_{r}(r \geqslant 1)$ which are self-adjoint with respect to $\|\cdot\|_{0,0}$ on $\mathscr{C}_{0}^{\infty}\left(\mathbb{R}^{2 n}, \mathbf{E}_{x_{0}}\right)$, and

$$
\begin{aligned}
L_{2}^{0}= & \mathscr{L}_{0}-2 \omega_{d, x_{0}}, \\
\underline{\mathcal{Q}}_{1}= & \mathcal{O}_{1}-\pi \sqrt{-1}\left\langle\left(\nabla_{\mathcal{R}}^{X} \mathbf{J}\right)_{x_{0}} e_{l}, e_{m}\right\rangle c\left(e_{l}\right) c\left(e_{m}\right)+\left(\partial_{\mathcal{R}} \tau\right)_{x_{0}}, \\
\underline{\mathcal{Q}}_{2}= & \mathcal{O}_{2}-R_{x_{0}}^{\mathrm{Cliff}}\left(\mathcal{R}, e_{l}\right) \nabla_{0, e_{l}}-\frac{\pi}{2} \sqrt{-1}\left\langle\left(\nabla^{X} \nabla^{X} \mathbf{J}\right)_{(\mathcal{R}, \mathcal{R}), x_{0}} e_{l}, e_{m}\right\rangle c\left(e_{l}\right) c\left(e_{m}\right) \\
& +\frac{1}{2}\left(R_{x_{0}}^{E}+\frac{1}{2} \operatorname{Tr}\left[R_{x_{0}}^{T^{(1,0)} X}\right]\right)\left(e_{l}, e_{m}\right) c\left(e_{l}\right) c\left(e_{m}\right)+\sum_{|\alpha|=2}\left(\partial^{\alpha} \tau\right)_{x_{0}} \frac{Z^{\alpha}}{\alpha !}+\frac{1}{4} r_{x_{0}}^{X} .
\end{aligned}
$$

such that

$$
L_{2}^{t}=L_{2}^{0}+\sum_{r=1}^{\infty} \underline{\mathcal{Q}}_{r} t^{r} .
$$

Proof. Set $g_{i j}(Z)=g^{T X}\left(e_{i}, e_{j}\right)(Z)=\left\langle e_{i}, e_{j}\right\rangle_{Z}$ and let $\left(g^{i j}(Z)\right)$ be the inverse of the matrix $\left(g_{i j}(Z)\right)$. By [4, Proposition 1.28] (cf. [15, Lemma 4.5]), we have

$$
\begin{aligned}
& g_{i j}(Z)=\delta_{i j}+\frac{1}{3}\left\langle R_{x_{0}}^{T X}\left(\mathcal{R}, e_{i}\right) \mathcal{R}, e_{j}\right\rangle_{x_{0}}+\mathscr{O}\left(|Z|^{3}\right), \\
& \kappa(Z)=\left|\operatorname{det}\left(g_{i j}(Z)\right)\right|^{1 / 2}=1+\frac{1}{6}\left\langle R_{x_{0}}^{T X}\left(\mathcal{R}, e_{i}\right) \mathcal{R}, e_{i}\right\rangle_{x_{0}}+\mathscr{O}\left(|Z|^{3}\right) .
\end{aligned}
$$

If $\Gamma_{i j}^{l}$ is the connection form of $\nabla^{T X}$ with respect to the basis $\left\{e_{i}\right\}$, we have $\left(\nabla_{e_{i}}^{T X} e_{j}\right)(Z)=$ $\Gamma_{i j}^{l}(Z) e_{l}$. Owing to (2.8) (cf. [24, (1.32)]),

$$
\Gamma_{i j}^{l}(Z)=\frac{1}{3}\left\langle R_{x_{0}}^{T X}\left(\mathcal{R}, e_{j}\right) e_{i}+R_{x_{0}}^{T X}\left(\mathcal{R}, e_{i}\right) e_{j}, e_{l}\right\rangle_{x_{0}}+\mathscr{O}\left(|Z|^{2}\right) .
$$

Let $\Gamma^{E}, \Gamma^{L}, \Gamma^{\text {Cliff }}$ be the connection forms of $\nabla^{E}, \nabla^{L}, \nabla^{\text {Cliff }}$ with respect to any fixed frames for $E, L, \Lambda\left(T^{*(0,1)} X\right)$ which are parallel along the curve $\gamma_{Z}$ under our trivializations on $B^{T_{x_{0}} X}(0, \varepsilon)$. By [4, Proposition 1.18] the Taylor coefficients of $\Gamma \cdot\left(e_{j}\right)(Z)$ at $x_{0}$ to order $r$ are only determined by those of $R^{\bullet}$ to order $r-1$, and

$$
\sum_{|\alpha|=r}\left(\partial^{\alpha} \Gamma^{\bullet}\right)_{x_{0}}\left(e_{j}\right) \frac{Z^{\alpha}}{\alpha !}=\frac{1}{r+1} \sum_{|\alpha|=r-1}\left(\partial^{\alpha} R^{\bullet}\right)_{x_{0}}\left(\mathcal{R}, e_{j}\right) \frac{Z^{\alpha}}{\alpha !} .
$$

(2.4), (2.10) yield on $B^{T_{x_{0}} X}(0, \varepsilon / t)$,

$$
\begin{aligned}
\left.\nabla_{t, e_{i}}\right|_{Z}= & \kappa^{\frac{1}{2}}(t Z)\left[\nabla_{e_{i}}+\left(\frac{1}{t} \Gamma^{L}\left(e_{i}\right)+t \Gamma^{E}\left(e_{i}\right)+t \Gamma^{\mathrm{Cliff}}\left(e_{i}\right)\right)(t Z)\right] \kappa^{-\frac{1}{2}}(t Z) \\
= & \kappa^{\frac{1}{2}}(t Z)\left[\nabla_{e_{i}}+\left(\frac{1}{2} R_{x_{0}}^{L}+\frac{t}{3}\left(\partial_{k} R^{L}\right)_{x_{0}} Z_{k}\right.\right. \\
& \left.\left.\quad+\frac{t^{2}}{4} \sum_{|\alpha|=2}\left(\partial^{\alpha} R^{L}\right)_{x_{0}} \frac{Z^{\alpha}}{\alpha !}+\frac{t^{2}}{2} R_{x_{0}}^{E}+\frac{t^{2}}{2} R_{x_{0}}^{\text {Cliff }}\right)\left(\mathcal{R}, e_{i}\right)+\mathscr{O}\left(t^{3}\right)\right] \kappa^{-\frac{1}{2}}(t Z) .
\end{aligned}
$$

By the definition of $\nabla^{\text {Cliff }}$, for $Y, U \in \mathscr{C}^{\infty}(X, T X)$,

$$
\left[\nabla_{U}^{\text {Cliff }}, c(Y)\right]=c\left(\nabla_{U}^{T X} Y\right) .
$$


For $\psi \in T^{*} X \otimes \operatorname{End}\left(\Lambda\left(T^{*(0,1)} X\right)\right) \simeq T^{*} X \otimes\left(C(T X) \otimes_{\mathbb{R}} \mathbb{C}\right)$, where $C(T X)$ is the Clifford algebra bundle of $T X$, we still denote by $\nabla^{X} \psi$ the covariant derivative of $\psi$ induced by $\nabla^{T X}$. By using (2.12), we observe that

$$
\begin{aligned}
\nabla_{Y}^{X}\left(\psi\left(\widetilde{e}_{j}\right) c\left(\widetilde{e}_{j}\right)\right) & \left.=\left(\nabla_{Y}^{X} \psi\right)\left(\widetilde{e}_{j}\right) c\left(\widetilde{e}_{j}\right)+\psi\left(\nabla_{Y}^{T X} \widetilde{e}_{j}\right) c\left(\widetilde{e}_{j}\right)+\psi\left(\widetilde{e}_{j}\right) c\left(\nabla_{Y}^{T X} \widetilde{e}_{j}\right)\right) \\
& =\left(\nabla_{Y}^{X} \psi\right)\left(\widetilde{e}_{j}\right) c\left(\widetilde{e}_{j}\right) .
\end{aligned}
$$

Thus for $k \geqslant 2$,

$$
\begin{aligned}
& \left(R^{L}\left(\widetilde{e}_{l}, \widetilde{e}_{m}\right) c\left(\widetilde{e}_{l}\right) c\left(\widetilde{e}_{m}\right)\right)(t Z)= \\
& =\left.\sum_{r=0}^{k} \frac{\partial^{r}}{\partial t^{r}}\left[\left(R^{L}\left(\widetilde{e}_{l}, \widetilde{e}_{m}\right) c\left(\widetilde{e}_{l}\right) c\left(\widetilde{e}_{m}\right)\right)(t Z)\right]\right|_{t=0} \frac{t^{r}}{r !}+\mathscr{O}\left(t^{k+1}\right) \\
& =\left(R_{x_{0}}^{L}+t\left(\nabla_{\mathcal{R}}^{X} R^{L}\right)_{x_{0}}+\frac{t^{2}}{2}\left(\nabla^{X} \nabla^{X} R^{L}\right)_{(\mathcal{R}, \mathcal{R}), x_{0}}\right)\left(e_{l}, e_{m}\right) c\left(e_{l}\right) c\left(e_{m}\right)+\mathscr{O}\left(t^{3}\right) .
\end{aligned}
$$

Owing to (1.4), (2.4), (2.11) and (2.13)-(2.14),

$$
\begin{aligned}
L_{2}^{t}= & -g^{i j}(t Z)\left(\nabla_{t, e_{i}} \nabla_{t, e_{j}}-t \Gamma_{i j}^{l}(t Z) \nabla_{t, e_{l}}\right)+\frac{t^{2}}{4} r^{X}(t Z) \\
& +\frac{1}{2}\left\{\left[R^{L}+t^{2}\left(R^{E}+\frac{1}{2} \operatorname{Tr}\left[R^{T^{(1,0)} X}\right]\right)\right]\left(\widetilde{e}_{l}, \widetilde{e}_{m}\right) c\left(\widetilde{e}_{l}\right) c\left(\widetilde{e}_{m}\right)\right\}(t Z) \\
& =-g^{i j}(t Z)\left(\nabla_{t, e_{i}} \nabla_{t, e_{j}}-t \Gamma_{i j}^{l}(t Z) \nabla_{t, e_{l}}\right) \\
& +\frac{1}{2}\left(R_{x_{0}}^{L}+t\left(\nabla_{\mathcal{R}}^{X} R^{L}\right)_{x_{0}}+\frac{t^{2}}{2}\left(\nabla^{X} \nabla^{X} R^{L}\right)_{(\mathcal{R}, \mathcal{R}), x_{0}}\right)\left(e_{l}, e_{m}\right) c\left(e_{l}\right) c\left(e_{m}\right) \\
& +\frac{t^{2}}{2}\left(R_{x_{0}}^{E}+\frac{1}{2} \operatorname{Tr}\left[R_{x_{0}}^{T^{(1,0)} X}\right]\right)\left(e_{l}, e_{m}\right) c\left(e_{l}\right) c\left(e_{m}\right)+\frac{t^{2}}{4} r_{x_{0}}^{X}+\mathscr{O}\left(t^{3}\right) .
\end{aligned}
$$

Note that $\nabla^{X} g^{T X}=0$. Comparing (1.5), (2.8), (2.11), (2.15), with [24, (1.37)], we get (2.7).

To prove the self-adjointness of $L_{2}^{0}$, and $\underline{\mathcal{Q}}_{r}(r \geqslant 1)$ with respect to $\|\cdot\|_{0,0}$ on $\mathscr{C}_{0}^{\infty}\left(\mathbb{R}^{2 n}, \mathbf{E}_{x_{0}}\right)$, we observe that it follows from the fact that $L_{2}^{t}$ is self-adjoint with respect to $\|\cdot\|_{0,0}$ on $\mathscr{C}_{0}^{\infty}\left(B^{T_{x_{0}} X}(0, \varepsilon / t), \mathbf{E}_{x_{0}}\right)$.

Let $P^{N}, \underline{P}^{N}$ be the orthogonal projections from $\left(L^{2}\left(\mathbb{R}^{2 n}, \mathbf{E}_{x_{0}}\right),\|\|_{0,0}\right)$ onto $N=$ $\operatorname{Ker} \mathscr{L}_{0}$, Ker $L_{2}^{0}$, and let $P^{N}\left(Z, Z^{\prime}\right), \underline{P}^{N}\left(Z, Z^{\prime}\right)$ be the smooth kernel of $P^{N}, \underline{P}^{N}$ with respect to $d v_{T X}(Z)$. Set $P^{N^{\perp}}=\mathrm{Id}-P^{N}, \underline{P}^{N^{\perp}}=\mathrm{Id}-\underline{P}^{N}$. Recall that $q=0$ in (1.6), thus $\omega_{d} \leq-\mu_{0}$ on $\Lambda^{>0}\left(T^{*(0,1)} X\right)$, by (2.6),

$$
\underline{P}^{N}\left(Z, Z^{\prime}\right)=P^{N}\left(Z, Z^{\prime}\right) I_{\mathbb{C} \otimes E} .
$$

Theorem 2.3. We have the relation

$$
\underline{P}^{N} \underline{Q}_{1} \underline{P}^{N}=0 .
$$

Proof. By [24, (1.92), (1.94), (1.96)], we have

$$
\begin{aligned}
& \left(\nabla_{U}^{X} R^{L}\right)(V, W)=-2 \pi \sqrt{-1}\left\langle\left(\nabla_{U}^{X} \mathbf{J}\right) V, W\right\rangle, \\
& \left(\nabla_{\mathcal{R}} \tau\right)_{x_{0}}=-2 \pi \sqrt{-1}\left\langle\left(\nabla_{\mathcal{R}}^{X} \mathbf{J}\right) w_{i}, \bar{w}_{i}\right\rangle, \\
& P^{N} \mathcal{O}_{1} P^{N}=0 .
\end{aligned}
$$


FIRST COEFFICIENTS OF THE ASYMPTOTIC EXPANSION OF THE BERGMAN KERNEL 15 By (2.6), (2.16) and (2.18), we get (2.17).

Set

$$
\begin{aligned}
F_{2}= & \left(L_{2}^{0}\right)^{-1} \underline{P}^{N^{\perp}} \underline{\mathcal{Q}}_{1}\left(L_{2}^{0}\right)^{-1} \underline{P}^{N^{\perp}} \underline{\mathcal{Q}}_{1} \underline{P}^{N}-\left(L_{2}^{0}\right)^{-1} \underline{P}^{N^{\perp}} \underline{\mathcal{Q}}_{2} \underline{P}^{N} \\
& +\underline{P}^{N} \underline{\mathcal{Q}}_{1}\left(L_{2}^{0}\right)^{-1} \underline{P}^{N^{\perp}} \underline{\mathcal{Q}}_{1}\left(L_{2}^{0}\right)^{-1} \underline{P}^{N^{\perp}}-\underline{P}^{N} \underline{\mathcal{Q}}_{2}\left(L_{2}^{0}\right)^{-1} \underline{P}^{N^{\perp}} \\
& +\underline{P}^{N^{\perp}}\left(L_{2}^{0}\right)^{-1} \underline{\mathcal{Q}}_{1} \underline{P}^{N} \underline{\mathcal{Q}}_{1}\left(L_{2}^{0}\right)^{-1} \underline{P}^{N^{\perp}}-\underline{P}^{N} \underline{\mathcal{Q}}_{1} \underline{P}^{N^{\perp}}\left(L_{2}^{0}\right)^{-2} \underline{\mathcal{Q}}_{1} \underline{P}^{N}
\end{aligned}
$$

Then by Theorem 2.3 and the same argument as in $[24, \S 1.5,1.6]$, we get

$$
\boldsymbol{b}_{1}\left(x_{0}\right)=F_{2}(0,0) \text {. }
$$

By Theorem 2.2, the third and fourth terms in (2.19) are adjoint of the first two terms, thus we only need to compute the first two terms and the last two terms in (2.19).

2.3. Computing the coefficient $b_{1}$. From now on, we assume that $\mathbf{J}=J$. By [24, (2.13)],

$$
\begin{aligned}
& \nabla_{U}^{X} J \text { is skew-adjoint and the tensor }\left\langle\left(\nabla^{X} J\right) \cdot, \cdot\right\rangle \text { is of the type } \\
& \left(T^{*(1,0)} X\right)^{\otimes 3} \oplus\left(T^{*(0,1)} X\right)^{\otimes 3} .
\end{aligned}
$$

In what follows we will use the complex coordinates $z=\left(z_{1}, \cdots, z_{n}\right)$, such that $w_{i}=\sqrt{2} \frac{\partial}{\partial z_{i}}$ is an orthonormal basis of $T_{x_{0}}^{(1,0)} X$. Then $Z=z+\bar{z}$ and will also identify $z$ to $\sum_{i} z_{i} \frac{\partial}{\partial z_{i}}$ and $\bar{z}$ to $\sum_{i} \bar{z}_{i} \frac{\partial}{\partial \bar{z}_{i}}$ when we consider $z$ and $\bar{z}$ as vector fields. Remark that

$$
\left|\frac{\partial}{\partial z_{i}}\right|^{2}=\left|\frac{\partial}{\partial \bar{z}_{i}}\right|^{2}=\frac{1}{2}, \quad \text { so that }|z|^{2}=|\bar{z}|^{2}=\frac{1}{2}|Z|^{2} .
$$

It is very useful to rewrite $\mathscr{L}_{0}$ by using the creation and annihilation operators. Set

$$
b_{i}=-2 \nabla_{0, \frac{\partial}{\partial z_{i}}}=-2 \frac{\partial}{\partial z_{i}}+\pi \bar{z}_{i}, \quad b_{i}^{+}=2 \nabla_{0, \frac{\partial}{\partial \bar{z}_{i}}}=2 \frac{\partial}{\partial \bar{z}_{i}}+\pi z_{i}, \quad b=\left(b_{1}, \cdots, b_{n}\right) .
$$

Then for any polynomial $g(z, \bar{z})$ on $z$ and $\bar{z}$,

$$
\begin{aligned}
& {\left[b_{i}, b_{j}^{+}\right]=b_{i} b_{j}^{+}-b_{j}^{+} b_{i}=-4 \pi \delta_{i j},} \\
& {\left[b_{i}, b_{j}\right]=\left[b_{i}^{+}, b_{j}^{+}\right]=0,} \\
& {\left[g(z, \bar{z}), b_{j}\right]=2 \frac{\partial}{\partial z_{j}} g(z, \bar{z}), \quad\left[g(z, \bar{z}), b_{j}^{+}\right]=-2 \frac{\partial}{\partial \bar{z}_{j}} g(z, \bar{z}) .}
\end{aligned}
$$

As $\mathbf{J}=J, a_{j}=2 \pi$ in (1.7). By (2.5) and (2.6) (cf. [24, (2.23)]),

$$
\begin{aligned}
& \mathcal{O}_{1}=-\frac{4 \pi \sqrt{-1}}{3} b_{i}\left\langle\left(\nabla_{\bar{z}}^{X} J\right) \bar{z}, \frac{\partial}{\partial \bar{z}_{i}}\right\rangle+\frac{4 \pi \sqrt{-1}}{3}\left\langle\left(\nabla_{z}^{X} J\right) z, \frac{\partial}{\partial z_{i}}\right\rangle b_{i}^{+}, \\
& \mathscr{L}_{0}=b_{j} b_{j}^{+}, \quad L_{2}^{0}=b_{j} b_{j}^{+}+4 \pi \bar{w}^{j} \wedge i_{\bar{w}_{j}} .
\end{aligned}
$$

We found the following result in [24, Theorem 1.15]: 
Theorem 2.4. The spectrum of the restriction of $\mathscr{L}_{0}$ on $L^{2}\left(\mathbb{R}^{2 n}\right)$ is given by

$$
\left.\operatorname{Spec} \mathscr{L}_{0}\right|_{L^{2}\left(\mathbb{R}^{2 n}\right)}=\left\{4 \pi \sum_{i=1}^{n} \alpha_{i}: \alpha=\left(\alpha_{1}, \cdots, \alpha_{n}\right) \in \mathbb{N}^{n}\right\}
$$

and an orthogonal basis of the eigenspace of $4 \pi \sum_{i=1}^{n} \alpha_{i}$ is given by

$$
b^{\alpha}\left(z^{\beta} \exp \left(-\frac{\pi}{2} \sum_{i}\left|z_{i}\right|^{2}\right)\right), \quad \text { with } \beta \in \mathbb{N}^{n} .
$$

From (2.27), we get

$$
P^{N}\left(Z, Z^{\prime}\right)=\exp \left(-\frac{\pi}{2} \sum_{i}\left(\left|z_{i}\right|^{2}+\left|z_{i}^{\prime}\right|^{2}-2 z_{i} \bar{z}_{i}^{\prime}\right)\right) .
$$

As $\mathbf{J}=J$ here, we know the function $\tau$ therein is $2 \pi n$. By (2.6), (2.21)-(2.23),

$$
\begin{aligned}
\underline{\mathcal{Q}}_{1}=\mathcal{O}_{1} & -2 \pi \sqrt{-1}\left[\left\langle\left(\nabla_{\bar{z}}^{X} J\right) \frac{\partial}{\partial \bar{z}_{l}}, \frac{\partial}{\partial \bar{z}_{m}}\right\rangle d \bar{z}_{l} d \bar{z}_{m}+4\left\langle\left(\nabla_{z}^{X} J\right) \frac{\partial}{\partial z_{l}}, \frac{\partial}{\partial z_{m}}\right\rangle i_{\frac{\partial}{\partial \bar{z}_{l}}}{ }^{\frac{\partial}{\partial \bar{z}_{m}}}\right], \\
\underline{\mathcal{Q}}_{2}=\mathcal{O}_{2} & +R_{x_{0}}^{\text {Cliff }}\left(\mathcal{R}, \frac{\partial}{\partial \bar{z}_{i}}\right) b_{i}-R_{x_{0}}^{\text {Cliff }}\left(\mathcal{R}, \frac{\partial}{\partial z_{i}}\right) b_{i}^{+} \\
& -\frac{\pi}{2} \sqrt{-1}\left\langle\left(\nabla^{X} \nabla^{X} J\right)_{(\mathcal{R}, \mathcal{R})} e_{l}, e_{m}\right\rangle c\left(e_{l}\right) c\left(e_{m}\right) \\
& +\frac{1}{2}\left(R_{x_{0}}^{E}+\frac{1}{2} \operatorname{Tr}\left[R_{x_{0}}^{T^{(1,0)} X}\right]\right)\left(e_{l}, e_{m}\right) c\left(e_{l}\right) c\left(e_{m}\right)+\frac{1}{4} r_{x_{0}}^{X} .
\end{aligned}
$$

Recall that by [24, (1.98), (2.24)], (2.23),

$$
b_{i}^{+} P^{N}=0, \quad\left(b_{i} P^{N}\right)\left(Z, Z^{\prime}\right)=2 \pi\left(\bar{z}_{i}-\bar{z}_{i}^{\prime}\right) P^{N}\left(Z, Z^{\prime}\right), \quad\left(\mathcal{O}_{1} P^{N}\right)(Z, 0)=0 .
$$

By (2.16), (2.21), (2.25), (2.28), (2.29) and (2.30), as in [24, (2.24)],

$$
\begin{aligned}
\left(\underline{\mathcal{Q}}_{1} \underline{P}^{N}\right)\left(Z, Z^{\prime}\right)= & {\left[-\frac{2 \sqrt{-1}}{3} b_{i} b_{j}\left\langle\left(\nabla_{\frac{\partial}{\partial \bar{z}} \bar{z}_{j}}^{X} J\right) \bar{z}^{\prime}, \frac{\partial}{\partial \bar{z}_{i}}\right\rangle-\frac{4 \pi \sqrt{-1}}{3} b_{i}\left\langle\left(\nabla_{\bar{z}^{\prime}}^{X} J\right) \bar{z}^{\prime}, \frac{\partial}{\partial \bar{z}_{i}}\right\rangle\right.} \\
& \left.-\sqrt{-1}\left\langle\left(\nabla_{\frac{\partial}{\partial \bar{z}_{k}}}^{X} J\right) \frac{\partial}{\partial \bar{z}_{l}}, \frac{\partial}{\partial \bar{z}_{m}}\right\rangle d \bar{z}_{l} d \bar{z}_{m}\left(b_{k}+2 \pi \bar{z}_{k}^{\prime}\right)\right] P^{N}\left(Z, Z^{\prime}\right) I_{\mathbb{C} \otimes E} .
\end{aligned}
$$

Thus by Theorem 2.4 and relations (2.21), (2.25) and (2.31),

$$
\begin{aligned}
\left(\left(L_{2}^{0}\right)^{-1} \underline{P}^{N^{\perp}} \underline{\mathcal{Q}}_{1} \underline{P}^{N}\right) & \left(Z, Z^{\prime}\right)=-\sqrt{-1}\left[\frac{b_{i} b_{j}}{12 \pi}\left\langle\left(\nabla_{\frac{\partial}{\partial \bar{z}_{j}}}^{X} J\right) \bar{z}^{\prime}, \frac{\partial}{\partial \bar{z}_{i}}\right\rangle+\frac{b_{i}}{3}\left\langle\left(\nabla_{\bar{z}^{\prime}}^{X} J\right) \bar{z}^{\prime}, \frac{\partial}{\partial \bar{z}_{i}}\right\rangle\right. \\
+ & \left.\left\langle\left(\nabla_{\frac{\partial}{\partial \bar{z}_{k}}}^{X} J\right) \frac{\partial}{\partial \bar{z}_{l}}, \frac{\partial}{\partial \bar{z}_{m}}\right\rangle d \bar{z}_{l} d \bar{z}_{m}\left(\frac{b_{k}}{12 \pi}+\frac{\bar{z}_{k}^{\prime}}{4}\right)\right] P^{N}\left(Z, Z^{\prime}\right) I_{\mathbb{C} \otimes E} .
\end{aligned}
$$

By (2.21), (2.30) and (2.32),

$$
\begin{aligned}
& \left(\underline{P}^{N^{\perp}}\left(L_{2}^{0}\right)^{-1} \underline{\mathcal{Q}}_{1} \underline{P}^{N}\right)\left(0, Z^{\prime}\right)=-\frac{\sqrt{-1}}{12}\left\langle\left(\nabla_{\bar{z}^{\prime}}^{X} J\right) \frac{\partial}{\partial \bar{z}_{l}}, \frac{\partial}{\partial \bar{z}_{m}}\right\rangle d \bar{z}_{l} d \bar{z}_{m} P^{N}\left(0, Z^{\prime}\right) I_{\mathbb{C} \otimes E}, \\
& \left(\underline{P}^{N^{\perp}}\left(L_{2}^{0}\right)^{-1} \underline{\mathcal{Q}}_{1} \underline{P}^{N}\right)(Z, 0)=-\frac{\sqrt{-1}}{6}\left\langle\left(\nabla_{\bar{z}}^{X} J\right) \frac{\partial}{\partial \bar{z}_{l}}, \frac{\partial}{\partial \bar{z}_{m}}\right\rangle d \bar{z}_{l} d \bar{z}_{m} P^{N}(Z, 0) I_{\mathbb{C} \otimes E} .
\end{aligned}
$$


FIRST COEFFICIENTS OF THE ASYMPTOTIC EXPANSION OF THE BERGMAN KERNEL 17

Recall that $L_{2}^{0}, \mathcal{Q}_{1}$ are self-adjoint with respect to $\|\cdot\|_{0,0}$ on $\mathscr{C}_{0}^{\infty}\left(\mathbb{R}^{2 n}, \mathbf{E}_{x_{0}}\right)$. After taken the adjoint of (2.33), by (2.22), we get

$$
\begin{aligned}
& \left(\underline{P}^{N} \underline{\mathcal{Q}}_{1}\left(L_{2}^{0}\right)^{-1} \underline{P}^{N^{\perp}}\right)\left(Z^{\prime}, 0\right)=\frac{\sqrt{-1}}{3}\left\langle\left(\nabla_{z^{\prime}}^{X} J\right) \frac{\partial}{\partial z_{l}}, \frac{\partial}{\partial z_{m}}\right\rangle I_{\mathbb{C} \otimes E^{i}} \frac{\partial}{\partial \bar{z}_{m}}{ }^{i} \frac{\partial}{\partial \bar{z}_{l}} P^{N}\left(Z^{\prime}, 0\right), \\
& \left(\underline{P}^{N} \underline{\mathcal{Q}}_{1}\left(L_{2}^{0}\right)^{-1} \underline{P}^{N^{\perp}}\right)(0, Z)=\frac{2 \sqrt{-1}}{3 \pi}\left\langle\left(\nabla_{z}^{X} J\right) \frac{\partial}{\partial z_{l}}, \frac{\partial}{\partial z_{m}}\right\rangle I_{\mathbb{C} \otimes E^{i}}{\frac{\partial}{\partial \bar{z}_{m}}}^{i} \frac{\partial}{\partial \bar{z}_{l}} P^{N}(0, Z) .
\end{aligned}
$$

Note that $\int_{\mathbb{C}}|z|^{2} e^{-\pi|z|^{2}}=1 / \pi$. By (2.21), (2.22), (2.28), (2.33) and (2.34),

$$
\begin{aligned}
& \left(\underline{P}^{N^{\perp}}\left(L_{2}^{0}\right)^{-1} \underline{\mathcal{Q}}_{1} \underline{P}^{N} \underline{\mathcal{Q}}_{1}\left(L_{2}^{0}\right)^{-1} \underline{P}^{N^{\perp}}\right)(0,0) \\
& \quad=\frac{1}{36 \pi}\left\langle\left(\nabla_{\frac{\partial}{\partial \bar{z}_{k}}}^{X} J\right) \frac{\partial}{\partial \bar{z}_{l}}, \frac{\partial}{\partial \bar{z}_{m}}\right\rangle\left\langle\left(\nabla_{\frac{\partial}{\partial z_{k}}}^{X} J\right) \frac{\partial}{\partial z_{i}}, \frac{\partial}{\partial z_{j}}\right\rangle d \bar{z}_{l} d \bar{z}_{m} I_{\mathbb{C} \otimes E^{i}}{\frac{\partial}{\partial \bar{z}_{j}}}^{i} \frac{\partial}{\partial \bar{z}_{i}}, \\
& -\left(\underline{P}^{N} \underline{\mathcal{Q}}_{1} \underline{P}^{N^{\perp}}\left(L_{2}^{0}\right)^{-2} \underline{\mathcal{Q}}_{1} \underline{P}^{N}\right)(0,0) \\
& =-\frac{1}{9 \pi}\left\langle\left(\nabla_{\frac{\partial}{\partial z_{k}}}^{X} J\right) \frac{\partial}{\partial z_{i}}, \frac{\partial}{\partial z_{j}}\right\rangle\left\langle\left(\nabla_{\frac{\partial}{\partial \bar{z}_{k}}}^{X} J\right) \frac{\partial}{\partial \bar{z}_{l}}, \frac{\partial}{\partial \bar{z}_{m}}\right\rangle I_{\mathbb{C} \otimes E^{i}}{\frac{\partial}{\partial \bar{z}_{j}}}^{i} \frac{\partial}{\partial \bar{z}_{i}} d \bar{z}_{l} d \bar{z}_{m} I_{\mathbb{C} \otimes E} \\
& =-\frac{1}{9 \pi}\left\langle\left(\nabla_{\frac{\partial}{\partial \bar{z}_{k}}}^{X} J\right) \frac{\partial}{\partial \bar{z}_{l}},\left(\nabla_{\frac{\partial}{\partial z_{k}}}^{X} J\right) \frac{\partial}{\partial z_{l}}\right\rangle .
\end{aligned}
$$

Let $h_{i}(Z)$ (resp. $F(Z)$ ) be polynomials in $Z$ with degree 1 (resp. 2), then by Theorem 2.4, (2.24), (2.25) and (2.30),

$$
\begin{aligned}
& \left(\mathscr{L}_{0}^{-1} P^{N^{\perp}} h_{i} b_{i} P^{N}\right)(0,0)=\left(\mathscr{L}_{0}^{-1} P^{N^{\perp}} b_{i} h_{i} P^{N}\right)(0,0)=-\frac{1}{2 \pi} \frac{\partial h_{i}}{\partial z_{i}}, \\
& \left(\mathscr{L}_{0}^{-1} P^{N^{\perp}} F P^{N}\right)(0,0)=-\frac{1}{4 \pi^{2}} \frac{\partial^{2} F}{\partial z_{i} \partial \bar{z}_{i}}, \\
& \left(\left(L_{2}^{0}\right)^{-1} F d \bar{z}_{l} d \bar{z}_{m} \underline{P}^{N}\right)(0,0)=\frac{1}{2 \pi}\left(\left(L_{2}^{0}\right)^{-1} \frac{\partial^{2} F}{\partial z_{i} \partial \bar{z}_{i}}\left(b_{i} z_{i}+2\right) d \bar{z}_{l} d \bar{z}_{m} \underline{P}^{N}\right)(0,0) \\
& \quad=\frac{1}{8 \pi^{2}} \frac{\partial^{2} F}{\partial z_{i} \partial \bar{z}_{i}}\left(\left(\frac{b_{i} z_{i}}{3}+1\right) d \bar{z}_{l} d \bar{z}_{m} \underline{P}^{N}\right)(0,0)=\frac{1}{24 \pi^{2}} \frac{\partial^{2} F}{\partial z_{i} \partial \bar{z}_{i}} d \bar{z}_{l} d \bar{z}_{m} I_{\mathbb{C} \otimes E} .
\end{aligned}
$$

Observe that for a monomial $Q$ in $b_{i}, z_{i}, b_{i}^{+}, \bar{z}_{i}$, if the total degree of $b_{i}, z_{i}$ is not the same as the total degree of $b_{i}^{+}, \bar{z}_{i}$, then $\left(Q \underline{P}^{N}\right)(0,0)=0$. Theorem 2.4 and relations (2.22), (2.25), (2.29), (2.30), (2.33) and (2.37) imply that

$$
\begin{aligned}
\left(\left(L_{2}^{0}\right)^{-1} \underline{P}^{N^{\perp}}\right. & \left.\underline{\mathcal{Q}}_{1}\left(L_{2}^{0}\right)^{-1} \underline{\mathcal{Q}}_{1} \underline{P}^{N}\right)(0,0)=\left(\left(L_{2}^{0}\right)^{-1} \underline{P}^{N^{\perp}}(-8 \pi \sqrt{-1})\left\langle\left(\nabla_{z}^{X} J\right) \frac{\partial}{\partial z_{i}}, \frac{\partial}{\partial z_{j}}\right\rangle\right. \\
& \left.i_{\frac{\partial}{\partial \bar{z}_{i}}} i \frac{\partial}{\partial \bar{z}_{j}} \times \frac{-\sqrt{-1}}{6}\left\langle\left(\nabla_{\bar{z}}^{X} J\right) \frac{\partial}{\partial \bar{z}_{l}}, \frac{\partial}{\partial \bar{z}_{m}}\right\rangle d \bar{z}_{l} d \bar{z}_{m} P^{N}\right)(0,0) I_{\mathbb{C} \otimes E} \\
= & \frac{4 \pi}{3}\left(\left(L_{2}^{0}\right)^{-1} \underline{P}^{N^{\perp}}\left\langle\left(\nabla_{z}^{X} J\right) \frac{\partial}{\partial z_{l}},\left(\nabla_{\bar{z}}^{X} J\right) \frac{\partial}{\partial \bar{z}_{l}}\right\rangle P^{N}\right)(0,0) I_{\mathbb{C} \otimes E} \\
= & -\frac{1}{3 \pi}\left\langle\left(\nabla_{\frac{\partial}{\partial z_{k}}}^{X} J\right) \frac{\partial}{\partial z_{l}},\left(\nabla_{\frac{\partial}{\partial \bar{z}_{k}}}^{X} J\right) \frac{\partial}{\partial \bar{z}_{l}}\right\rangle I_{\mathbb{C} \otimes E} .
\end{aligned}
$$


Now we will compute $\left(\left(L_{2}^{0}\right)^{-1} \underline{P}^{N^{\perp}} \underline{\mathcal{Q}}_{2} \underline{P}^{N}\right)(0,0)$. By (1.1) and (2.21),

$$
\operatorname{Tr}\left[R^{T^{(1,0)} X}\right]=2\left\langle R^{T X} \frac{\partial}{\partial z_{j}}, \frac{\partial}{\partial \bar{z}_{j}}\right\rangle+\frac{1}{2}\left\langle\left(\nabla_{\frac{\partial}{\partial z_{l}}}^{X} J\right) \frac{\partial}{\partial z_{j}},\left(\nabla_{\frac{\partial}{\partial \bar{z}_{m}}}^{X} J\right) \frac{\partial}{\partial \bar{z}_{j}}\right\rangle d \bar{z}_{m} \wedge d z_{l} .
$$

By definition, for $U, V \in T X$, we have

$$
\left(\nabla^{X} \nabla^{X} J\right)_{(U, V)}-\left(\nabla^{X} \nabla^{X} J\right)_{(V, U)}=\left[R^{T X}(U, V), J\right] .
$$

By [24, (2.19)], for $u_{1}, u_{2}, u_{3} \in T^{(1,0)} X, \bar{v}_{1}, \bar{v}_{2} \in T^{(0,1)} X$,

$$
\begin{aligned}
& \left(\nabla^{X} \nabla^{X} J\right)_{\left(u_{1}, u_{2}\right)} u_{3},\left(\nabla^{X} \nabla^{X} J\right)_{\left(\bar{u}_{1}, \bar{u}_{2}\right)} u_{3} \in T^{(0,1)} X, \quad\left(\nabla^{X} \nabla^{X} J\right)_{\left(\bar{u}_{1}, u_{2}\right)} \bar{u}_{3} \in T^{(0,1)} X \\
& 2 \sqrt{-1}\left\langle\left(\nabla^{X} \nabla^{X} J\right)_{\left(u_{1}, \bar{v}_{1}\right)} u_{2}, \bar{v}_{2}\right\rangle=\left\langle\left(\nabla_{u_{1}}^{X} J\right) u_{2},\left(\nabla_{\bar{v}_{1}}^{X} J\right) \bar{v}_{2}\right\rangle .
\end{aligned}
$$

By (1.1), (2.16), (2.28), (2.29), (2.30) and (2.39),

$$
\begin{aligned}
\underline{P}^{N^{\perp}}\left(\underline{\mathcal{Q}}_{2}\right. & \left.-\mathcal{O}_{2}\right) \underline{P}^{N}=\underline{P}^{N^{\perp}}\left\{\frac{1}{2} \operatorname{Tr}\left[R^{T^{(1,0)} X}\right]\left(\mathcal{R}, \frac{\partial}{\partial \bar{z}_{i}}\right) b_{i}\right. \\
& -\left\langle\left(R^{T X}\left(\mathcal{R}, \frac{\partial}{\partial \bar{z}_{i}}\right) b_{i}-2 \pi \sqrt{-1}\left(\nabla^{X} \nabla^{X} J\right)_{(\mathcal{R}, \mathcal{R})}\right) \frac{\partial}{\partial z_{l}}, \frac{\partial}{\partial \bar{z}_{l}}\right\rangle \\
& +\left\langle\left(\frac{1}{2} R^{T X}\left(\mathcal{R}, \frac{\partial}{\partial \bar{z}_{i}}\right) b_{i}-\pi \sqrt{-1}\left(\nabla^{X} \nabla^{X} J\right)_{(\mathcal{R}, \mathcal{R})}\right) \frac{\partial}{\partial \bar{z}_{l}}, \frac{\partial}{\partial \bar{z}_{m}}\right\rangle d \bar{z}_{l} d \bar{z}_{m} \\
& \left.+\left(R^{E}+\frac{1}{2} \operatorname{Tr}\left[R^{T^{(1,0)} X}\right]\right)\left(\frac{\partial}{\partial \bar{z}_{l}}, \frac{\partial}{\partial \bar{z}_{m}}\right) d \bar{z}_{l} d \bar{z}_{m}\right\} \underline{P}^{N} .
\end{aligned}
$$

Thus by (2.30), (2.37), (2.40) and (2.42),

$$
\begin{aligned}
- & \left(\left(L_{2}^{0}\right)^{-1} \underline{P}^{N^{\perp}}\left(\underline{\mathcal{Q}}_{2}-\mathcal{O}_{2}\right) \underline{P}^{N}\right)(0,0)=\left\{\frac{1}{4 \pi} \operatorname{Tr}\left[R^{T^{(1,0)} X}\right]\left(\frac{\partial}{\partial z_{i}}, \frac{\partial}{\partial \bar{z}_{i}}\right)\right. \\
& -\frac{1}{2 \pi}\left\langle R^{T X}\left(\frac{\partial}{\partial z_{i}}, \frac{\partial}{\partial \bar{z}_{i}}\right)-\sqrt{-1}\left(2\left(\nabla^{X} \nabla^{X} J\right)_{\left(\frac{\partial}{\partial z_{i}}, \frac{\partial}{\partial \bar{z}_{i}}\right)}-\left[R^{T X}\left(\frac{\partial}{\partial z_{i}}, \frac{\partial}{\partial \bar{z}_{i}}\right), J\right]\right) \frac{\partial}{\partial z_{l}}, \frac{\partial}{\partial \bar{z}_{l}}\right\rangle \\
& -\frac{1}{24 \pi}\left\langle R^{T X}\left(\frac{\partial}{\partial z_{i}}, \frac{\partial}{\partial \bar{z}_{i}}\right)-\sqrt{-1}\left(2\left(\nabla^{X} \nabla^{X} J\right)_{\left(\frac{\partial}{\partial \bar{z}_{i}}, \frac{\partial}{\partial z_{i}}\right)}+\left[R^{T X}\left(\frac{\partial}{\partial z_{i}}, \frac{\partial}{\partial \bar{z}_{i}}\right), J\right]\right) \frac{\partial}{\partial \bar{z}_{l}}, \frac{\partial}{\partial \bar{z}_{m}}\right\rangle d \bar{z}_{l} d \bar{z}_{m} \\
& \left.-\frac{1}{8 \pi}\left(R^{E}+\frac{1}{2} \operatorname{Tr}\left[R^{T^{(1,0)} X}\right]\right)\left(\frac{\partial}{\partial \bar{z}_{l}}, \frac{\partial}{\partial \bar{z}_{m}}\right) d \bar{z}_{l} d \bar{z}_{m}\right\} I_{\mathbb{C} \otimes E} .
\end{aligned}
$$


From (2.39), (2.41) and (2.43), we have

(2.44)

$$
\begin{aligned}
-( & \left.\left(L_{2}^{0}\right)^{-1} \underline{P}^{N^{\perp}}\left(\underline{\mathcal{Q}}_{2}-\mathcal{O}_{2}\right) \underline{P}^{N}\right)(0,0)= \\
= & \left\{\frac{1}{4 \pi} \operatorname{Tr}\left[R^{T^{(1,0)} X}\right]\left(\frac{\partial}{\partial z_{i}}, \frac{\partial}{\partial \bar{z}_{i}}\right)-\frac{1}{2 \pi}\left\langle\left(R^{T X}\left(\frac{\partial}{\partial z_{i}}, \frac{\partial}{\partial \bar{z}_{i}}\right)-2 \sqrt{-1}\left(\nabla^{X} \nabla^{X} J\right)\left(\frac{\partial}{\partial z_{i}}, \frac{\partial}{\partial \bar{z}_{i}}\right)\right) \frac{\partial}{\partial z_{l}}, \frac{\partial}{\partial \bar{z}_{l}}\right\rangle\right. \\
& \left.+\left[\frac{1}{24 \pi}\left\langle R^{T X}\left(\frac{\partial}{\partial z_{i}}, \frac{\partial}{\partial \bar{z}_{i}}\right) \frac{\partial}{\partial \bar{z}_{l}}, \frac{\partial}{\partial \bar{z}_{m}}\right\rangle-\frac{1}{8 \pi}\left(R^{E}+\frac{1}{2} \operatorname{Tr}\left[R^{T^{(1,0)} X}\right]\right)\left(\frac{\partial}{\partial \bar{z}_{l}}, \frac{\partial}{\partial \bar{z}_{m}}\right)\right] d \bar{z}_{l} d \bar{z}_{m}\right\} I_{\mathbb{C} \otimes E} \\
= & \left\{\frac{3}{8 \pi}\left\langle\left(\nabla_{\frac{\partial}{\partial z_{l}}}^{X} J\right) \frac{\partial}{\partial z_{j}},\left(\nabla_{\frac{\partial}{\partial \bar{z}_{l}}}^{X} J\right) \frac{\partial}{\partial \bar{z}_{j}}\right\rangle-\frac{1}{12 \pi}\left\langle R^{T X}\left(\frac{\partial}{\partial \bar{z}_{l}}, \frac{\partial}{\partial \bar{z}_{m}}\right) \frac{\partial}{\partial z_{i}}, \frac{\partial}{\partial \bar{z}_{i}}\right\rangle d \bar{z}_{l} d \bar{z}_{m}\right. \\
& \left.-\frac{1}{8 \pi} R^{E}\left(\frac{\partial}{\partial \bar{z}_{l}}, \frac{\partial}{\partial \bar{z}_{m}}\right) d \bar{z}_{l} d \bar{z}_{m}\right\} I_{\mathbb{C} \otimes E .}
\end{aligned}
$$

By [24, (2.39)],

$$
-\left(\mathscr{L}_{0}^{-1} P^{N^{\perp}} \mathcal{O}_{2} \underline{P}^{N}\right)(0,0)=\frac{1}{2 \pi}\left\{\left\langle R^{T X}\left(\frac{\partial}{\partial z_{i}}, \frac{\partial}{\partial \bar{z}_{j}}\right) \frac{\partial}{\partial z_{j}}, \frac{\partial}{\partial \bar{z}_{i}}\right\rangle+R^{E}\left(\frac{\partial}{\partial z_{i}}, \frac{\partial}{\partial \bar{z}_{i}}\right)\right\} I_{\mathbb{C} \otimes E}
$$

By (2.19), (2.20), (2.35), (2.36), (2.38), (2.44), (2.45), and the discussion after (2.20), we get

$$
\begin{aligned}
\boldsymbol{b}_{1}(x)= & \frac{1}{\pi}\left[\left\langle R^{T X}\left(\frac{\partial}{\partial z_{i}}, \frac{\partial}{\partial \bar{z}_{j}}\right) \frac{\partial}{\partial z_{j}}, \frac{\partial}{\partial \bar{z}_{i}}\right\rangle+R^{E}\left(\frac{\partial}{\partial z_{j}}, \frac{\partial}{\partial \bar{z}_{j}}\right)-\frac{1}{36} \sum_{k l}\left|\left(\nabla_{\frac{\partial}{\partial \bar{z}_{k}}}^{X} J\right) \frac{\partial}{\partial \bar{z}_{l}}\right|^{2}\right] I_{\mathbb{C} \otimes E} \\
& +\frac{1}{36 \pi}\left\langle\left(\nabla_{\frac{\partial}{\partial \bar{z}_{k}}}^{X} J\right) \frac{\partial}{\partial \bar{z}_{l}}, \frac{\partial}{\partial \bar{z}_{m}}\right\rangle\left\langle\left(\nabla_{\frac{\partial}{\partial z_{k}}}^{X} J\right) \frac{\partial}{\partial z_{i}}, \frac{\partial}{\partial z_{j}}\right\rangle d \bar{z}_{l} \wedge d \bar{z}_{m} I_{\mathbb{C} \otimes E} i_{\frac{\partial}{\partial \bar{z}_{j}}} \wedge i_{\frac{\partial}{\partial \bar{z}_{i}}} \\
& -\frac{1}{8 \pi}\left(\frac{2}{3}\left\langle R^{T X} \frac{\partial}{\partial z_{i}}, \frac{\partial}{\partial \bar{z}_{i}}\right\rangle+R^{E}\right)\left(\frac{\partial}{\partial \bar{z}_{l}}, \frac{\partial}{\partial \bar{z}_{m}}\right) d \bar{z}_{l} \wedge d \bar{z}_{m} I_{\mathbb{C} \otimes E} \\
& +\frac{1}{2 \pi}\left(\frac{2}{3}\left\langle R^{T X} \frac{\partial}{\partial z_{i}}, \frac{\partial}{\partial \bar{z}_{i}}\right\rangle+R^{E}\right)\left(\frac{\partial}{\partial z_{l}}, \frac{\partial}{\partial z_{m}}\right) I_{\mathbb{C} \otimes E} i_{\frac{\partial}{\partial \bar{z}_{m}}} \wedge i_{\frac{\partial}{\partial \bar{z}_{l}}} .
\end{aligned}
$$

Moreover, we learn from [24, Lemma 2.2] that

$$
r^{X}=-\left\langle R^{T X}\left(e_{i}, e_{j}\right) e_{i}, e_{j}\right\rangle=8\left\langle R^{T X}\left(\frac{\partial}{\partial z_{i}}, \frac{\partial}{\partial \bar{z}_{j}}\right) \frac{\partial}{\partial z_{j}}, \frac{\partial}{\partial \bar{z}_{i}}\right\rangle-\frac{1}{4}\left|\nabla^{X} J\right|^{2} .
$$

We are now ready to conclude. By (2.46) and (2.47), we get the formula (2.1) for $b_{1}$. We obtain then (2.2) by taking the trace of (2.1). The proof of Theorem 2.1 is complete.

\section{REFERENCES}

[1] A. Andreotti and H. Grauert, Théorème de finitude pour la cohomologie des espaces complexes, Bull. Soc. Math. France 90 (1962), 193-259.

[2] A. Andreotti and C. D. Hill, E. E. Levi convexity and the Hans Lewy problem. I. Reduction to vanishing theorems, Ann. Scuola Norm. Sup. Pisa (3) 26 (1972), 325-363.

[3] A. Andreotti and C. D. Hill, E. E. Levi convexity and the Hans Lewy problem. II. Vanishing theorems, Ann. Scuola Norm. Sup. Pisa (3) 26 (1972), 747-806.

[4] N. Berline, E. Getzler, and M. Vergne, Heat kernels and Dirac operators, Grundl. Math. Wiss. Band 298, Springer-Verlag, Berlin, 1992. 
[5] R. Berman and J. Sjöstrand, Asymptotics for Bergman-Hodge kernels for high powers of complex line bundles, Preprint available at arXiv:math.CV/0511158 (2005).

[6] J.-M. Bismut, Demailly's asymptotic Morse inequalities: a heat equation proof, J. Funct. Anal. 72 (1987), no. 2, 263-278.

[7] __ A local index theorem for non-Kähler manifolds, Math. Ann. 284 (1989), no. 4, 681699.

[8] _ Koszul complexes, harmonic oscillators, and the Todd class, J. Amer. Math. Soc. 3 (1990), no. 1, 159-256, With an appendix by the author and C. Soulé.

[9] J.-M. Bismut and É. Vasserot, The asymptotics of the Ray-Singer analytic torsion associated with high powers of a positive line bundle, Comm. Math. Phys. 125 (1989), no. 2, 355-367.

[10] D. Borthwick and A. Uribe, Almost complex structures and geometric quantization, Math. Res. Lett. 3 (1996), no. 6, 845-861. Erratum: 5 (1998),211-212.

[11] L. Boutet de Monvel, Hypoelliptic operators with double characteristics and related pseudodifferential operators, Comm. Pure Appl. Math. 27 (1974), 585-639.

[12] L. Boutet de Monvel and J. Sjöstrand, Sur la singularité des noyaux de Bergman et de Szegó, Journées Équations aux Dérivées Partielles de Rennes (1975), Soc. Math. France, Paris, 1976, pp. 123-164. Astérisque, No. 34-35.

[13] M. Braverman, Vanishing theorems on covering manifolds, Contemp. Math., vol. 231, Amer. Math. Soc., Providence, RI, 1999, pp. 1-23.

[14] D. Catlin, The Bergman kernel and a theorem of Tian, Analysis and geometry in several complex variables (Katata, 1997), Trends Math., Birkhäuser Boston, Boston, MA, 1999, pp. 1-23.

[15] X. Dai, K. Liu, and X. Ma, On the asymptotic expansion of Bergman kernel, C. R. Math. Acad. Sci. Paris 339 (2004), no. 3, 193-198. the full version: J. Differential Geom. to appear, math.DG/0404494

[16] J.-P. Demailly, Sur l'identité de Bochner-Kodaira-Nakano en gómétrie hermitienne, Lecture Notes in Math., vol. 1198, pp. 88-97, Springer Verlag, 1985.

[17] S. K. Donaldson, Remarks on gauge theory, complex geometry and 4-manifold topology, Fields Medallists' lectures, 384-403, World Sci. Ser. 20th Century Math., 5, World Sci. Publishing, River Edge, NJ, 1997.

[18] P. Gauduchon, Calabi's extremal Kähler metrics: an elementary introduction, book in preparation, 2005.

[19] Ph. A. Griffiths, The extension problem in complex analysis. II. Embeddings with positive normal bundle. Amer. J. Math. 88 (1966), 366-446.

[20] V. Guillemin and A. Uribe, The Laplace operator on the nth tensor power of a line bundle: eigenvalues which are uniformly bounded in n, Asymptotic Anal. 1 (1988), no. 2, 105-113.

[21] J. J. Kohn and H. Rossi, On the extension of holomorphic functions from the boundary of a complex manifold, Ann. of Math. (2) 81 (1965), 451-472

[22] Z. Lu, On the lower order terms of the asymptotic expansion of Tian-Yau-Zelditch, Amer. J. Math. 122 (2000), no. 2, 235-273.

[23] X. Ma and G. Marinescu, The Spin ${ }^{c}$ Dirac operator on high tensor powers of a line bundle, Math. Z. 240 (2002), no. 3, 651-664.

[24] _ Generalized Bergman kernels on symplectic manifolds, C. R. Math. Acad. Sci. Paris 339 (2004), no. 7, 493-498. The full version available at arXiv:math.DG/0411559

[25] __ Demailly's holomorphic Morse inequalities, Bergman kernels and applications, book in preparation, (2005).

[26] T. Ohsawa, Isomorphism theorems for cohomology groups of weakly 1-complete manifolds. Publ. Res. Inst. Math. Sci. 18 (1982), no. 1, 191-232.

[27] X. Wang, Canonical metric and stability of vector bundles over a projective manifold, Ph.D. thesis, Brandeis University, 2002. 
FIRST COEFFICIENTS OF THE ASYMPTOTIC EXPANSION OF THE BERGMAN KERNEL 21

[28] S. Zelditch, Szegó kernels and a theorem of Tian, Internat. Math. Res. Notices (1998), no. 6, 317-331.

Centre de Mathématiques Laurent Schwartz, UMr 7640 du CNRS, École PolyTeChnique, 91128 Palaiseau Cedex, France

$E$-mail address: ma@math.polytechnique.fr

Fachbereich Mathematik, Johann Wolfgang Goethe-Universität, Robert-MayerStrasse 10, 60054, Frankfurt am Main, Germany

E-mail address: marinesc@math.uni-frankfurt.de 